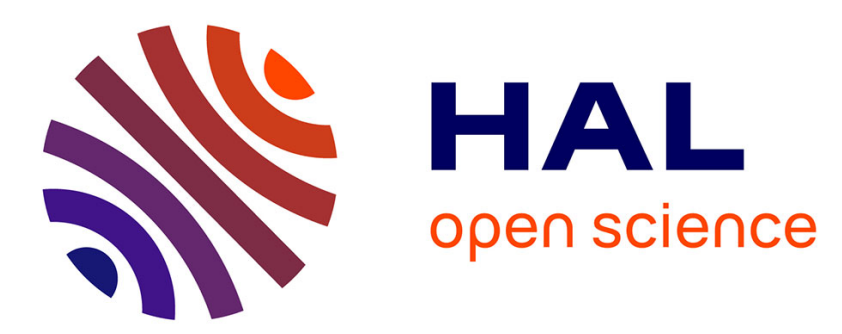

\title{
Multiscale thermomechanical modeling of shock-driven dry friction in hydrodynamics
}

\author{
Franck Dambakazi, Patrick Le Tallec, Jean-Philippe Perlat
}

\section{To cite this version:}

Franck Dambakazi, Patrick Le Tallec, Jean-Philippe Perlat. Multiscale thermomechanical modeling of shock-driven dry friction in hydrodynamics. Computer Methods in Applied Mechanics and Engineering, 2009, 198 (21-26), pp.1701-1715. 10.1016/j.cma.2008.12.019 . hal-00358907

\section{HAL Id: hal-00358907 https://hal.science/hal-00358907}

Submitted on 14 Jan 2020

HAL is a multi-disciplinary open access archive for the deposit and dissemination of scientific research documents, whether they are published or not. The documents may come from teaching and research institutions in France or abroad, or from public or private research centers.
L'archive ouverte pluridisciplinaire HAL, est destinée au dépôt et à la diffusion de documents scientifiques de niveau recherche, publiés ou non, émanant des établissements d'enseignement et de recherche français ou étrangers, des laboratoires publics ou privés. 


\title{
Multiscale thermomechanical modeling of shock-driven dry friction in hydrodynamics is
}

\author{
F. Dambakizi ${ }^{\mathrm{a}, \mathrm{b}}$, P. Le Tallec ${ }^{\mathrm{a}, *}$, J.P. Perlat ${ }^{\mathrm{b}}$ \\ a Ecole Polytechnique, Laboratoire de Mécanique des Solides, Palaiseau Route de Saclay, 91128 Palaiseau, France \\ ${ }^{\mathrm{b}}$ CEA/DAM, Commissariat à l'Enérgie Atomique, BP 12, 91680 Bruyères-le-Châtel, France
}

The purpose of the present work is to provide new insights in the understanding and computational modeling of shock-induced metal-on-metal dry friction. Based on a multiscale approach, we develop herein a 1D finite difference subgrid model. To adequately describe the physics of dynamic friction under shockinduced conditions, it accounts for frictional contact, elastoplastic yielding and work hardening, heating by friction and plastic work, thermal softening and melting, as well as dynamics effects. Temperature and dynamic elastoplasticity are predicted at a local scale through a nonlinear time implicit numerical solver. Two strategies have been considered for the coupling of the subgrid model to a standard thermoelastoplastic macroscopic model. The first one is velocity driven. Its implementation is rather straightforward, it leads to correct qualitative results but is restricted to sliding situations. To account for stick-slip cases, a second force driven downscaling strategy has been developed.

\section{Introduction}

Shock-driven dynamic friction refers to the physics that govern the shear stress acting along a material interface after the passage of a shock wave. It plays a key role in explosively driven systems where metal interfaces are submitted to relatively high sliding velocities and large contact pressures. It leads to complex interactions between the sliding bodies.

As observed by Oden [1] "dynamic friction is not a single phenomenon in itself but a collection of many complex thermomechanical phenomena whose features cannot be grasped through isolated simple experiments."

The sliding response of structures submitted to dynamic friction first depends on two parameters:

- $[v]$, the relative interfacial sliding velocity;

- $P$, the contact pressure applied at the interface.

\footnotetext{
is Expanded version of a talk presented at the USNCCM9 in honor of Prof. J. Tinsley Oden's 70th birthday (July 23-26, 2007 San Francisco, CA, USA).

* Corresponding author.

E-mail addresses: franck.dambakizi@cea.fr, franck.dambakizi@polytechnique edu (F. Dambakizi), patrick.letallec@polytechnique.fr (P. Le Tallec), jean-philippe. perlat@cea.fr (J.P. Perlat).
}

Depending on the values taken by both these parameters, the dynamic friction regimes can be sorted out from high-speed machining to ballistic penetration. Such a representation allows to differentiate the different experimental conditions that have been studied until now. In the past, dynamic friction with large slip velocities has been investigated under low contact pressures. In the pioneer works of Bowden and Freitag [3], Bowden and Persson [4] and Montgomery [5], the authors reported velocity weakening of the frictional shear stress as a function of increasing relative velocity. As a consequence much lower friction than expected was observed with a strong time dependence. On a physical point of view, the origin of this velocity weakening has been quickly attributed to thermal softening. It was shown that in the regime of high sliding velocity, the major part of the energy spent in overcoming the frictional resistance was appearing as heat near the interface. As the shock-induced sliding interfaces are brutally sheared, the rate of heat generation is much larger than it can be conducted away. This amount of heat causes thermal softening at the interface sub-surface (see Fig. 1), thus reducing the shearing strength and leading to lower values of frictional stress.

Recent experiments Juanicotena et al. [6], Ball et al. [7], have confirmed that severe loading conditions involving high contact pressures were also leading to extremely localized thermomechanical processes at the sliding interfaces: on a post-mortem observation under a microscope, they observe at the interface a layer of a few microns which has been severely heated and distorted. These 


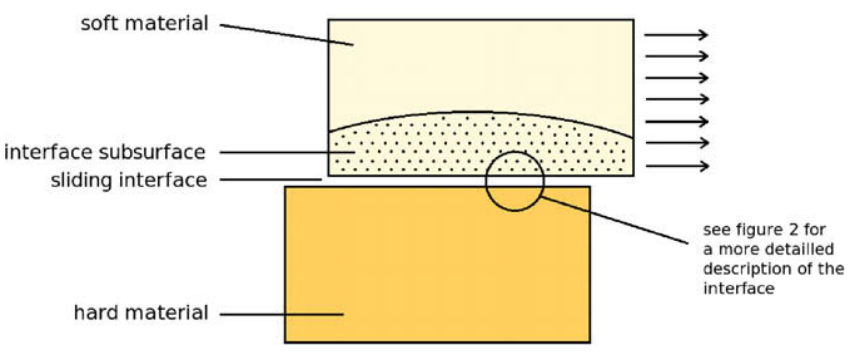

Fig. 1. Sketch of the contact region showing the areas of interest.

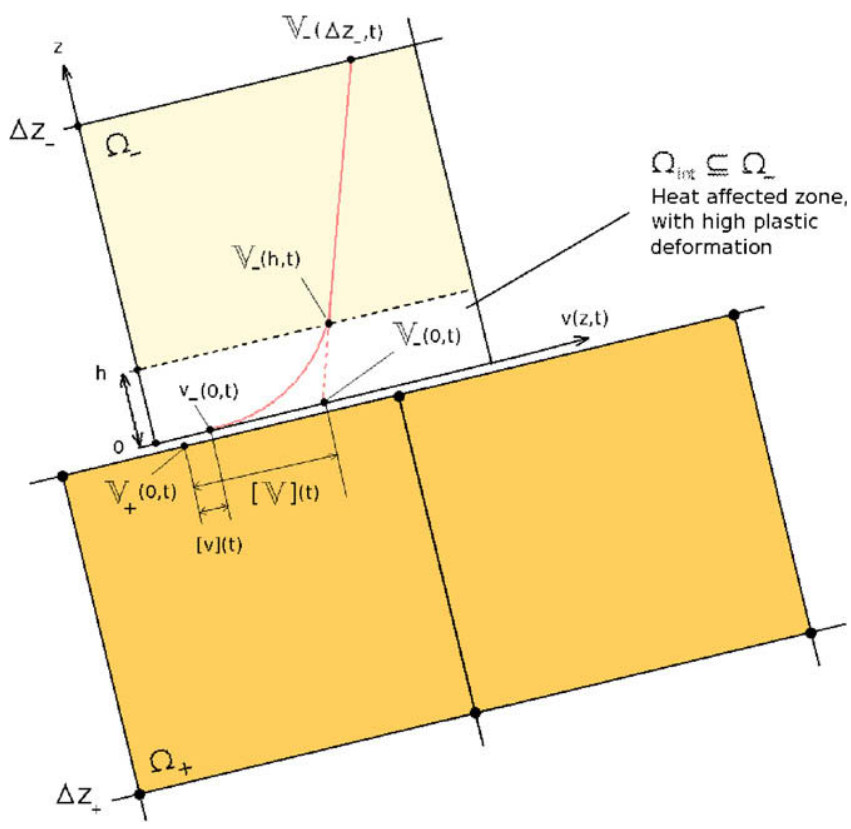

Fig. 2. Description of the interface at the macroscopic and microscopic scales.

local phenomena may profoundly change the state of interface during the dynamic slip process: on a very thin sheared layer $(\sim 0.1-5 \mu \mathrm{m})$ important strain rates are obtained with major irreversible plastic deformation $\varepsilon_{p}$.

Metal interfaces are also submitted to a warm-up phase due to frictional heat production, and plastic work. It leads to an important and localized rise in surface temperature $\Theta$, that may lead in extremely short time up to the fully melt temperature regime and to the formation of a thin molten metal film.

Modelling of the dynamic frictional behavior and the associated slip response therefore requires to focus on the thermomechanical response of the interfaces. Most numerical simulations are based on Coulomb's law of friction in which the frictional or tangential friction stress $\tau$ at the interface is directly proportional to the normal stress $\sigma_{n}$ and acts in the opposite direction of the sliding velocity $[v]$ :

$\tau=\mu\left|\sigma_{n}\right|$.

In Eq. (1), the thermomechanical behaviour of the sliding interface is described by a dimensionless scalar frictional coefficient $\mu$ that characterizes the interface. In the simplest models, this parameter is an empirical constant determined for each given pair of materials. To account for the velocity dependence of the effective shear stress, many numerical models have considered $\mu$ as a function of the sliding velocity $[v]$ often assumed to be monotonically weakening with increasing velocity: $\tau=\mu\left|\sigma_{n}\right|$,

$\mu\left([v], \mu_{0}, \mu_{\infty}, \vartheta\right)=\mu_{\infty}+\left(\mu_{0}-\mu_{\infty}\right) \exp ^{-\vartheta|| v] \mid}$.

This modified Coulomb's law of friction was shown to be in qualitative agreement with most of the experimental results. Nevertheless it requires to finely tune three parameters: the static friction coefficient $\mu_{0}$ that governs the low velocity behavior, the dynamic friction coefficient $\mu_{\infty}$ that controls the large relative velocity behavior, and the parameter $\vartheta>0$ that controls the rate of change of friction with changes in relative velocity. These coefficients turn out to be experience dependent. Moreover, there is no clear temperature's dependance in the model. Last, for the high pressure range and for systems of practical interest, the classical Coulomb's law of friction predicts a shear stress that greatly exceeds the Von Mises yield limit in pure shear $\left(\tau_{\max }=Y / \sqrt{3}\right)$, where $Y$ is the yield strength. As a consequence, Tresca's law of friction assuming that the frictional shear stress is limited by the yielding limit of the weaker material (Eq. (4)) seems more appropriate:

$\tau=\min \left(\mu\left|\sigma_{n}\right|, \frac{Y}{\sqrt{3}}\right)$

But all these attempts are not very satisfactory since they do not involve a clear relation between frictional stress, thermal softening and work hardening.

In order to compute the response of shock-induced friction systems under severe loading conditions, we therefore propose to develop a more detailed dynamic friction model. This model should account for the physical phenomena observed at the shock-induced sliding sub-surfaces (see Fig. 1), and be easily implemented in an hydrocode environment. Hydrocodes are specially designed for solving problems involving high energy, explosives, or shock propagation, situations where pressures waves and acceleration forces play a dominant role. They mostly use explicit time integration algorithms which means that the computational grids cannot be too fine. Based on a multiscale approach, we develop herein a 1D finite difference subgrid model. To adequately model the physics of dynamic friction under shock-induced conditions, it accounts for frictional contact, elastoplastic yielding and work hardening, heating by friction and plastic work, thermal softening and melting, as well as dynamics effects. The outline of the paper is as follows: Section 2 reviews the basic experimental framework at hand, Section 3 outlines the overall numerical strategy. The local model is detailed in Section 4. Sections 5 and 6 are devoted to the development and validation of adequate numerical coupling strategies.

\section{Experimental framework}

The loading regime considered in this work is described in Fig. 3 and corresponds to the area of interest, with velocities $[v]$ ranging from 100 to $1000 \mathrm{~m} \mathrm{~s}^{-1}$, and pressures taken between 5 and $100 \mathrm{GPa}$.

In addition to the reference experiments introduced in Section 1, two recent series of studies realized by Prakash et al. and Ball et al. must be mentioned. Irfan and Prakash [8] developed a plate-impact pressure-shear friction experiment. In these experiments interfacial slip speeds ranging from 1 to $60 \mathrm{~m} \mathrm{~s}^{-1}$, normal pressures of $1-3 \mathrm{GPa}$ and interfacial temperatures of $700{ }^{\circ} \mathrm{C}$ or more were obtained. For the studied cases, the general behaviour appears to be similar to what is observed at lighter loads. Thus, the coefficient of friction is found to decrease with increasing sliding speed. Nevertheless some important differences should be mentioned. For some loading conditions, they showed that with heavier loads the deformation of the solids appears to be primarily plastic. At higher speeds and even heavier loads, the frictional heating can become so severe that a continuous hydrodynamic 


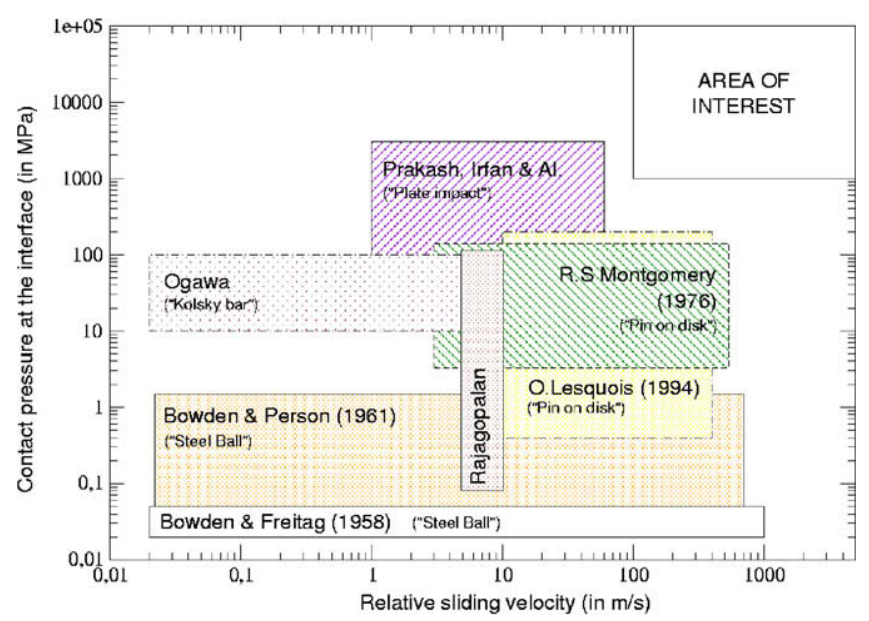

Fig. 3. Physical domain.

film of molten metal may appear at the interface within a very short time. To model the observed thermomechanical behavior, Prakash [8] recently developed a dynamic friction model where the resistance to motion is determined primarily by this molten film.

More recently, Ball et al. [7] developed a novel experimental technique for observing the sub-surface deformation in aluminium when sliding against a steel cylinder at high velocity and pressure (see Fig. 4). Associated with the aforementioned thermal heating and softening (see Fig. 5) they also observed the evolution of the microstructure in the vicinity of the sliding interface. Since the sheared layer is very thin $(\sim 0.1-5 \mu \mathrm{m})$ and the sliding speed is relatively high $\left(>100 \mathrm{~m} \mathrm{~s}^{-1}\right)$, important strain rates are obtained near the interface, resulting in major irreversible plastic deformation. In some cases, the interface sub-surface is sheared so rapidly that the global deformation is fully plastic.

Such regimes can be also observed in the dynamic friction experiment developed at the Cea/Dam by Juanicotena et al. [6]. Similar to the plate-impact technique described in [8], this experiment consists in studying the response of a cylindrical target when impacted by a metallic projectile.

This target is made up of two materials: a light central cone surrounded by an heavier confinement material (see Fig. 6). Such a geometry ensures the contact at the interface. Different values of contact pressures and sliding velocities can be attained by simply changing the nature of the flyer, the impact speed or the angle of the cone.

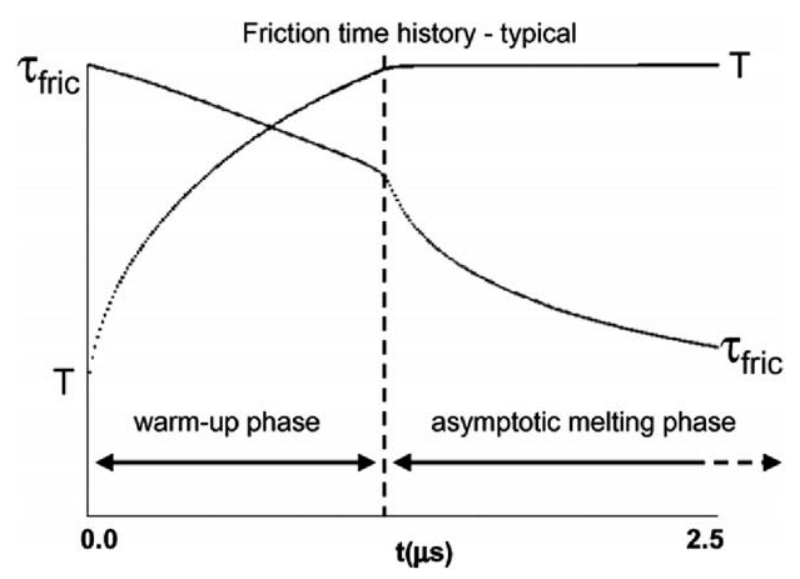

Fig. 5. Typical evolution of interface temperature and shear force as observed by Ball et al. [7].

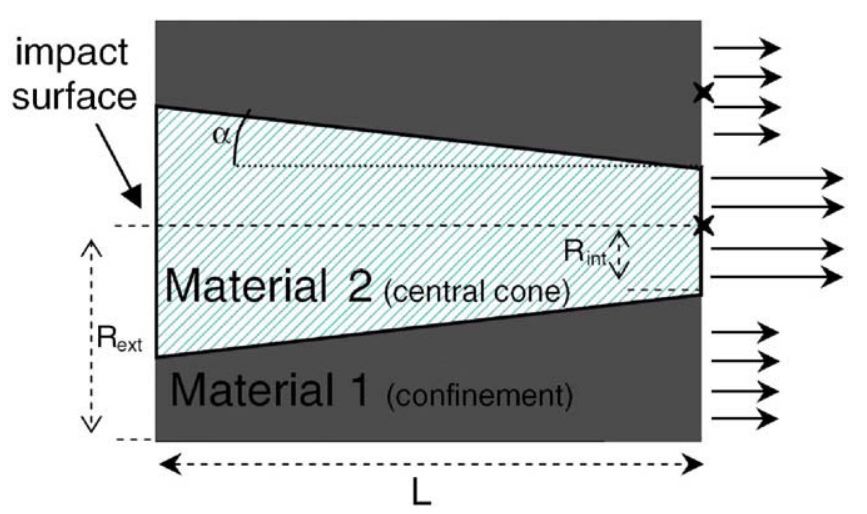

Fig. 6. CEA dynamic friction experiment setting: shock-induced motion of light material inside heavier one (from Juanicotena et al. [6]).

Upon striking the target, the projectile generates a plane shock wave that starts to propagate inside the tribopair materials. Because of its lower mechanical impedance, the central cone will first start to move. It results in a relative motion at the interface characterized by very large sliding velocities. To assess the magnitude of the slip response, the free surface velocity of the central cone is measured by laser interferometry for various given impact speeds. We may then express the interfacial slip response as a function of the loading conditions.

The observed results show that severe loading conditions lead to extremely localized thermomechanical processes. More precisely, we observe an important and localized increase in surface temperature due to frictional heat production. This thermal blowup (warm-up) leads in an extremely short time to the fully melt temperature regime and to the formation of a thin molten metal film. As a counter-effect, extreme shearing of the sub-surface generates large plastic deformations which tend to strengthen the material. Resulting interactions between both these phenomena are generally complex and may profoundly affect the state of the interface during the dynamic slip process.

These experimental studies do not completely characterize the shock-induced frictional properties in the high sliding velocity and high pressure regimes. This lack of data and the difficulties encountered in setting up concrete and physically based experiments could be overcome by using numerical simulations. Fig. 7 reports some classical output results obtained by hydrocodes when 


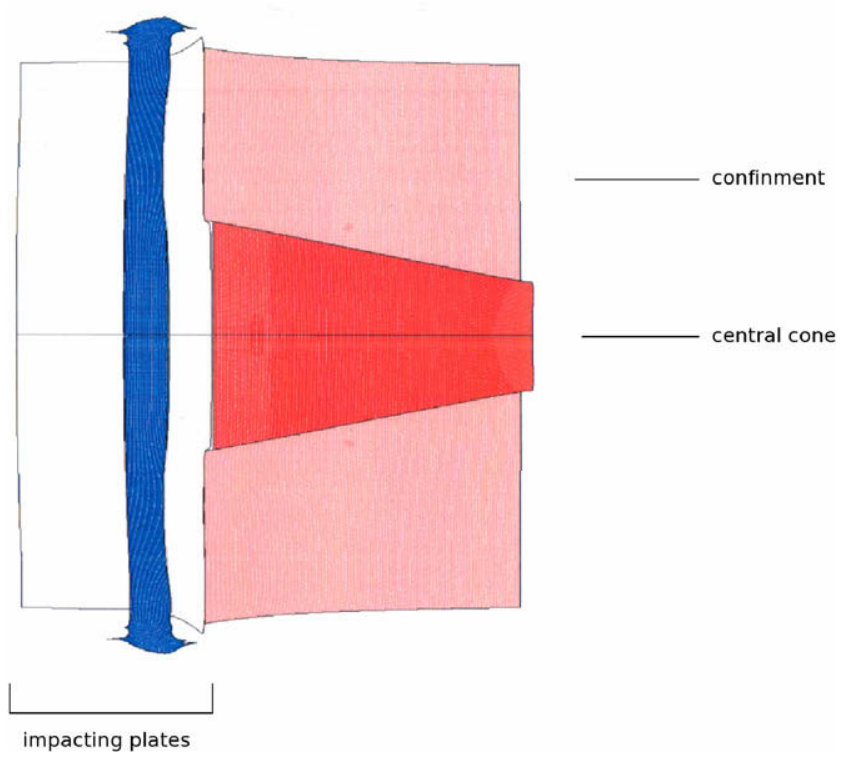

Fig. 7. Numerical solution of a typical dynamic friction experiment computed by an industrial hydrocode. The specifications are close to the experimental set up of Juanicotena et al. [6]. The dark center cone in aluminium is push ahead by the incoming wave and slides inside the center hole.

studying centimetric axisymmetric samples subjected to an impinging shock wave for a few microseconds.

The models use basic kinematically hardening and thermally softening elastoplastic laws in large strains and neglect heat conduction. The codes are explicit in time with FEM cell sizes of $10 \mu \mathrm{m}$ or more $(\Delta Z \sim 10 \mu \mathrm{m})$, and time steps of a few nanoseconds $\left(\Delta t \sim 1 \mathrm{e}^{-9} \mathrm{~s}\right)$. They either neglect friction or use simple empirical models (3) and (4) in which the frictional stress is related to the normal stress through modified Coulomb's laws:

$\tau=\min \left\{\left(\mu_{\infty}+\left(\mu_{0}-\mu_{\infty}\right) \cdot \exp ^{-\vartheta|[v]|}\right) \cdot \sigma_{n}, \mu_{T} \cdot \frac{Y\left(\Theta, \varepsilon_{p}\right)}{\sqrt{3}}\right\}$,

where $\mu_{T}$ is a user defined auxiliary friction coefficient. The center cone in aluminium is pushed ahead by the incoming wave and slides inside the steel hole.

Nevertheless, the observed predicted time histories are different from experiments even with these modified friction models. It could be explained by the lack of precision in computing frictional interfaces. Indeed, the FEM mesh cells used in classical hydrocodes fail to capture some key physical processes, such as interface heating and transient heat conduction which occur at lower scale. In addition, they do not track the thermomechanical history of the materials at the interface. The temperature $\Theta$ calculated in the friction law (5) generally corresponds to the average temperature value computed by the hydrocode at the center of a FEM interfacial cell. For extreme loading cases this average value may severely differ from the real interfacial temperature. It leads to an over-estimation of the interfacial shear stress implying more friction and therefore a limited relative motion. It is the same for the sub-surface elastoplastic deformation $\varepsilon_{p}$.

\section{Towards a physics-based multiscale numerical model: proposed strategies}

To improve the computational resolution of shock-driven interfaces we propose herein to develop a robust dynamic friction model for use in hydrocodes. This model requires that the physics of dynamic friction conditions is adequately modeled. Based on the 1D model developed by Ball et al. [7] it should therefore accounts

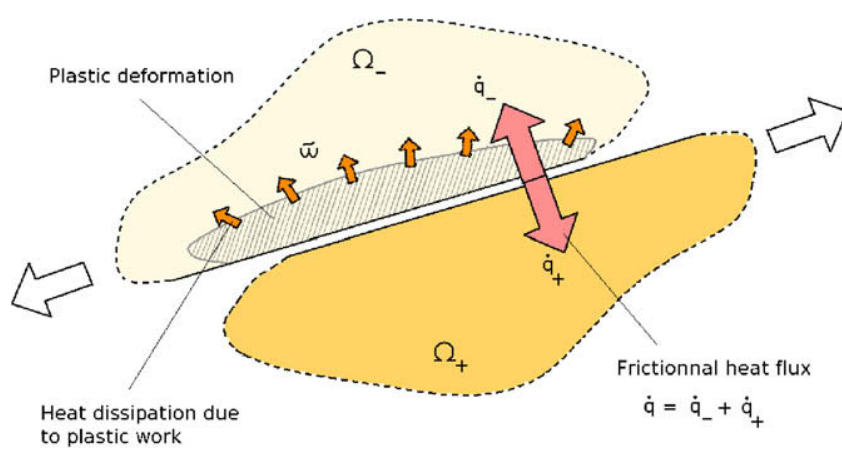

Fig. 8. The global geometry and the observed thermomechanical phenomena

for work hardening and thermal softening next to the frictional interface.

To model the slip response of shock-driven interfaces the dynamic friction problem is here treated as a two-body problem. As pictured in Fig. 8, it basically consists in studying the problem where a light metallic material subjected to normal contact pressures $\sigma_{n}=P$, denoted as body $\Omega_{-}$, slides at large relative velocity $[v]$ on an heavier metallic body, denoted $\Omega_{+}$.

\subsection{Modelling strategy}

From a numerical point of view, the main difficulties are related to the confinement of the thermomechanical processes over a thin layer of matter near the interface and to the construction of a numerical scheme which ensures that the interfacial temperature stays below the melting temperature of the sliding bodies.

In order to adequately capture these localized thermomechanical processes with computational cells, a multiscale approach is necessary (see Fig. 9). The subgrid model to be coupled to the existing macroscopic model should accurately resolve material interfaces and help to understand the dynamic frictional characteristics of sliding interfaces under relatively high sliding velocities, high normal pressures and elevated temperatures. The local model will therefore couple a detailed resolution of the heat equation in the interface layer taking into account the heat produced by friction as in $[9,10]$, and the thermal dependent elastoplastic model of this layer.

As pictured on Fig. 10, this multiscale approach uses three scales in space:

- Actual hydrocodes only consider the macroscopic scale where the light and heavy sliding materials, respectively, occupy the half spaces $\Omega_{-}=z \in\left[0, \Delta Z_{-}\right]$and $\Omega_{+}=z \in\left[\Delta Z_{+}, 0\right]$ (see Fig. 10) and where $\Delta Z_{-}$and $\Delta Z_{+}$are the size of the FEM cells in the soft and hard material, respectively.

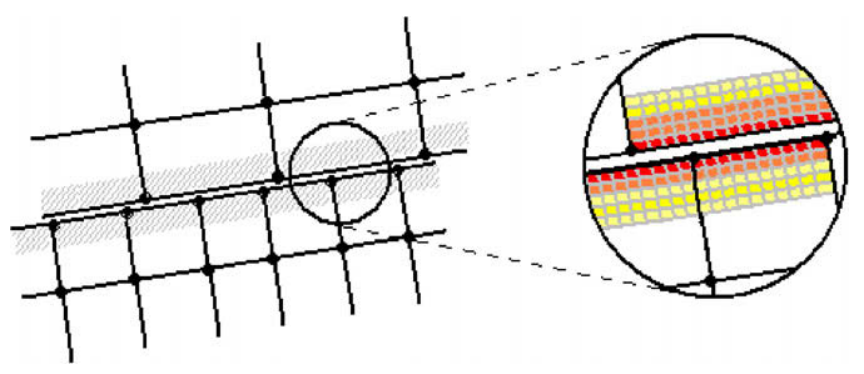

Fig. 9. Interfacial subgrid model accounting for sub-surface thermal softening and plastic work hardening. 


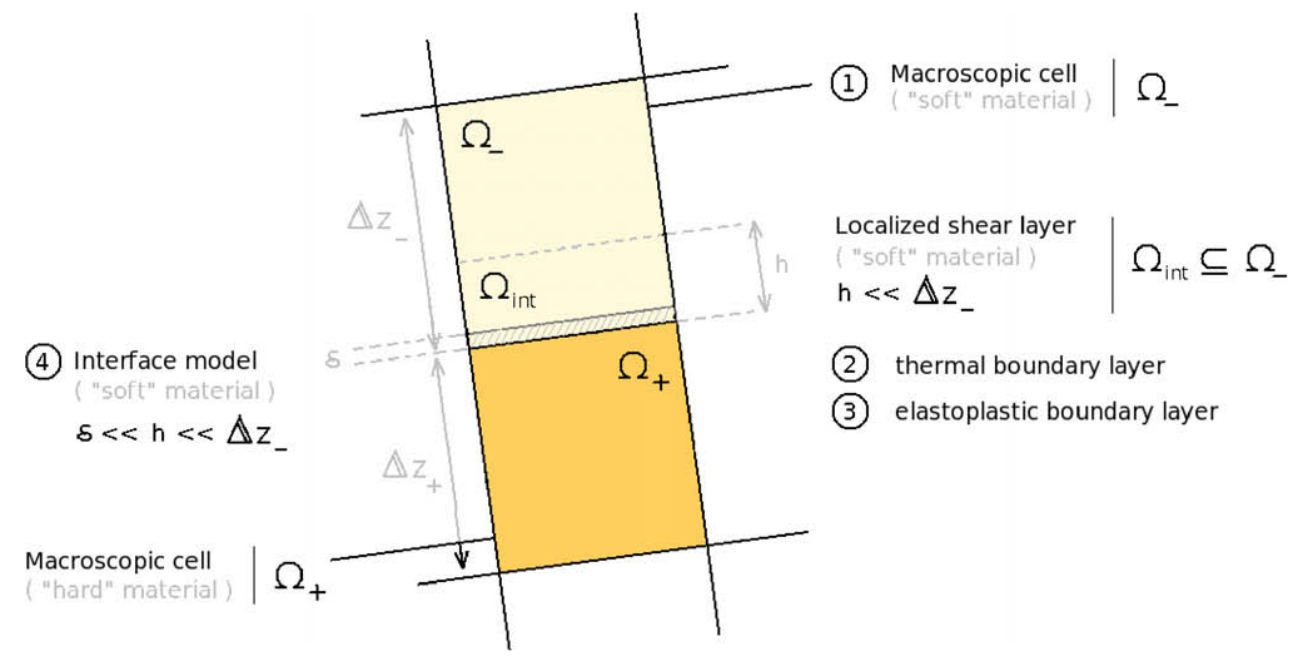

Fig. 10. Multiscale problem at the interface showing the hydrocode cells and the subgrid model.

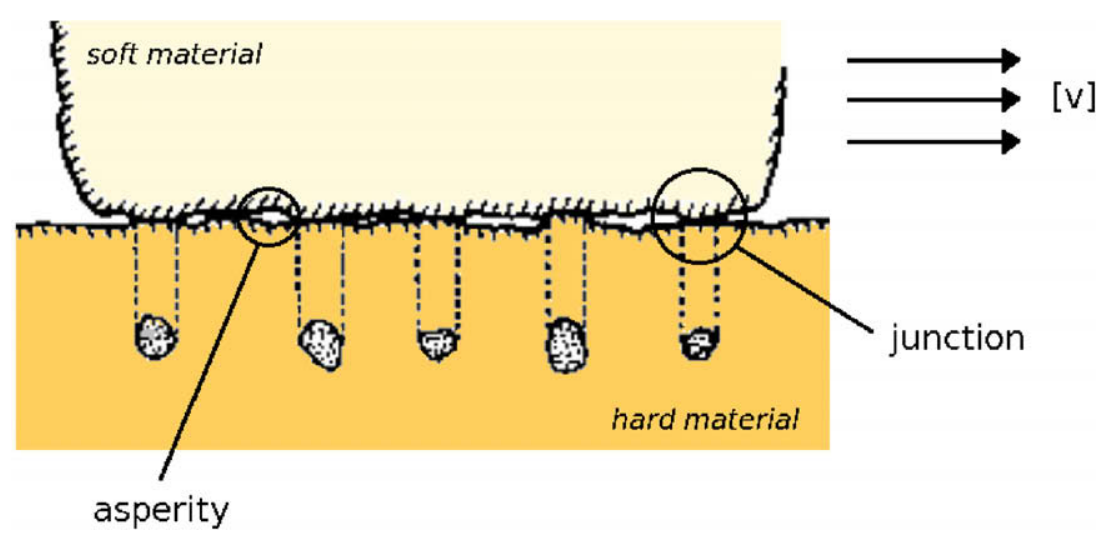

Fig. 11. Mechanical model including asperities and junctions at the sliding interface.

- At the local scale (microscopic scale) used in our subgrid model we suppose that shearing at the interface is confined in a thin layer of matter where all the key thermomechanical phenomena appear. Therefore, we define a subspace $\Omega_{\text {int }} \in \Omega_{-}$made of an heat affected zone (thermal boundary layer), and a plastic boundary layer.

- Finally, a nanoscopic scale might be considered (Fig. 11). In that case, the interface is described by a specific analytical model that accounts for surface irregularities such as asperities.

\subsection{Coupling strategy}

The efficiency of the local model strongly depends on the strategy that is defined to couple the local model to the global model (which is solved by the hydrocode). For the sake of simplicity we will differentiate the values computed at the macroscopic scale from the local values predicted by the subgrid model. As pictured in Fig. 2, the velocity values computed by the hydrocode are denoted by $\mathbb{V}$, whereas the real velocity field at microscopic scale is denoted by $v$. In the same way, the macroscopic shear stress is denoted by $\mathbb{T}$ and the real shear stress by $\tau$.

Downscale and upscale strategies consist in choosing adequate parameters that will be exchanged between the two scales. In the present study, two coupling methods have been considered. They basically consist in coupling downscale and upscale through far field shear stress $\mathbb{T}$ and macroscopic velocity fields $\mathbb{V}$.

\section{Multiscale thermomechanical model}

\subsection{Global model at the macroscopic scale}

The thermomechanical problem couples the stress tensor $\underline{\underline{\sigma}}(\underline{x}, t)$, the strain tensor $\underline{\varepsilon}(\underline{x}, t)$, the temperature field $\Theta(\underline{x}, t)$ and the material velocity $\underline{v}(\underline{x}, t)$. All of them are defined at a given material point $\underline{x}$, at a given instant $t$. They are governed by the standard equations of conservation of mass, momentum and energy coupled to an elastoplastic material model.

In conservative form, on any given material subdomain characterized by its reference configuration $\Omega_{0}$ or its present boundary $\Gamma(t)$, the conservative laws write:

$\frac{\mathrm{d}}{\mathrm{d} t} \int_{\Omega_{0}} \rho_{0} \mathrm{~d} \Omega=0$

$\frac{\mathrm{d}}{\mathrm{d} t} \int_{\Omega_{0}} \rho_{0} \cdot \underline{\mathbb{V}}(\underline{x}, t) \mathrm{d} \Omega=\int_{\Gamma(t)} \underline{\underline{\sigma}}(\underline{x}, t) \cdot \underline{n} \mathrm{~d} \Gamma$,

$\frac{\mathrm{d}}{\mathrm{d} t} \int_{\Omega_{0}} \rho_{0} \cdot\left(\frac{\underline{\mathbb{V}}^{2}(\underline{x}, t)}{2}+e\right) \mathrm{d} \Omega=\int_{\Gamma(t)} \underline{\mathbb{V}}(\underline{x}, t) \cdot \underline{\underline{\sigma}}(\underline{x}, t) \cdot \underline{n} \mathrm{~d} \Gamma$

Here, $e$ stands for the specific internal energy. In the present study it is assumed that the evolution of the deviatoric stress is given by $\underline{s}(\underline{x}, t)=\underline{\sigma}(\underline{x}, t)-P \underline{1 l}$, where $P$ is the hydrostatic pressure and $\underline{1 l}$ the identity tensor. The deviatoric stress then follows a standard law of elastoplasticity with constant shear modulus $G_{0}$, 
$\underline{\underline{\dot{s}}}(\underline{x}, t)=2 G_{0} \cdot \underline{\underline{d}}_{e}(\underline{x}, t)=2 G_{0}\left(\underline{\underline{d}}(\underline{x}, t)-\underline{\underline{d}}_{p}(\underline{x}, t)\right)$,

and with plastic strain evolution being governed by a Von Mises like flow rule:

$f(\underline{\underline{s}}, Y):=\sigma_{e q}-Y(\underline{x}, t) \leqslant 0$,

$\dot{\varepsilon}_{p}(\underline{x}, t) \cdot\left(\sigma_{e q}-Y(\underline{x}, t)\right)=0, \quad \dot{\varepsilon}_{p}(\underline{x}, t) \geqslant 0$,

$\underline{\underline{d}}_{p}(\underline{x}, t)=\frac{3}{2}\left(\dot{\varepsilon}_{p}(\underline{x}, t) \cdot \frac{\underline{s}}{\sigma_{e q}}, t\right)$.

Here $\sigma_{e q}$, the equivalent Von Mises stress is given by

$\sigma_{e q}=\sqrt{\frac{3}{2}}\|\underline{\underline{s}}(\underline{x}, t)\|$.

The SCG model [11] is used to characterize the evolution of the flow stress $Y$. This constitutive model takes into account work hardening, pressure effect, thermal softening and melting:

$Y\left(\varepsilon_{p}, G\right)=\max \left(Y_{\max }, Y_{0}\left(1+\beta \varepsilon_{p}\right)^{\eta}\right) \times \frac{G(P, \Theta)}{G_{0}}$,

$G(P, \Theta)=G_{0}\left(1+g P-h_{\Theta}(\Theta-300)\right) \times \exp \left(-\frac{0.001 \cdot \Theta}{\Theta_{F}-\Theta}\right)$.

Here, $Y_{0}$ is the reference yield strength of the local material, $P$ is the pressure, $\Theta$ the temperature, and $\beta, \eta, g, h_{\Theta}$ are material dependent parameters. It is to note that the first term on the right of Eq. (14) allows to model work hardening effects. The second term which is defined by Eq. (15) represents pressure hardening and thermal softening. The final exponential term was introduced by Ball et al. [7] and produces a rapid decay in flow stress as the melting temperature is approached.

The global model does not specify the constitutive law of the frictional interface. This will be introduced in the subgrid model at a spatial scale which is compatible with the local singularities of the temperature and plastic strain fields.

To compute the macroscopic behavior, both domains are discretized on a finite element grid and resolved by a locally developed explicit in time Lagrangian hydrocode [6]. Conservation of momentum is written on vertex based lagrangian cells. The conservation of mass and energy is imposed within each finite element.

\subsection{Specification of the local model at the microscopic scale}

At the microscopic scale and for the sheared layer configuration, we assume spatial homogeneity in the $x$ and $y$ directions, meaning that all field quantities defined in $\Omega_{\text {int }}$ depend on $(z, t)$ only.

As in [12], the local thermomechanical problem therefore couples:

- The deviatoric stress tensor $\underline{s}(z, t)$, that may be directly written as a function of the shear stress $\tau(z, t)$, so that:

$$
\underline{\underline{s}}(z, t)=\left(\begin{array}{ccc}
0 & 0 & \tau(z, t) \\
0 & 0 & 0 \\
\tau(z, t) & 0 & 0
\end{array}\right) .
$$

- The deviatoric strain tensor $\underline{\underline{d}}(z, t)$ which is written in terms of its elastic and plastic components, respectively, $\underline{\underline{d}}_{e}(z, t)$ and $\underline{\underline{d}}_{p}(z, t)$, and such that:

$$
\underline{\underline{d}}(z, t)=\underline{\underline{d}}_{e}(z, t)+\underline{\underline{d}}_{p}(z, t) \text {. }
$$

- The temperature field $\underline{\Theta}(z, t)$.

- The material velocity $\underline{v}(z, t)$.

They are all defined for a given material point $z \in \Omega_{\text {int }}=[0, h]$, and at each time $t$. They are governed by the energy and momen- tum conservation laws, by the elastoplastic constitutive law (9), by the flow rule (11), by the flow stress construction (14) and (15), and by a specific interface elastoplastic constitutive law to be specified below.

\subsection{Local energy conservation and transient heat problem}

As previously indicated, under shock loading conditions, the heat generated at the sliding interface dissipates into a very thin heat affected zone and decreases very quickly with distance from the interface. To model this thermal behaviour we use the transient model of conduction defined by Prakash [8]. This model assumes that both the material may be modeled by semi-infinite solids.

\subsubsection{Heat sources}

The heat generated during the slip process may be produced by two sources: on the one hand by the interfacial frictional work $\dot{q}(0, t)$ and on the other hand by plastic work in the sub-surface $\tilde{w}(z, t)$ which we will neglect for the time. The surfacic source $\dot{q}(0, t)$ is defined as a heat flow per unit time per unit area. If we assume that all the interfacial frictional work is converted to heat, we have:

$\dot{q}(0, t)=\beta_{T} \cdot \tau(0, t) \cdot[v](0, t)$.

The coefficient $\beta_{T}$ governs the partitioning of heat in the tribopair materials. Assuming a perfect contact, Vernotte [13] showed by equating the temperatures at the material interfaces that this coefficient was only dependent on the materials properties:

$\beta_{T}=\frac{k \sqrt{\alpha_{+}}}{k \sqrt{\alpha_{+}}+k_{+} \sqrt{\alpha}}$.

In Eq. (19), $k, \alpha, k_{+}$and $\alpha_{+}$are the thermal conductivity and thermal diffusivity of $\Omega_{i n t}$ and $\Omega_{+}$, respectively.

\subsubsection{Heat conduction equation}

The temperature increase for a given point $z \in \Omega_{\text {int }}$ is governed by the equation of conservation of energy, which in nonconservative form writes

$\frac{\partial^{2} \Theta(z, t)}{\partial z^{2}}-\frac{1}{\alpha} \frac{\partial \Theta(z, t)}{\partial t}=0$

For simplicity, we have taken here uniform diffusivity and conductivity coefficients $\alpha$ and $k$ inside the shear layer.

\subsubsection{Thermal boundary conditions}

In our case, we will suppose that at microscale the initial temperature field $\Theta(z, 0)$ is uniform in space, and equal to the postshock temperature $\Theta_{\text {ref }}$, such that:

$\Theta(z, 0)=\Theta_{\text {ref }}$.

The dissipation of heat at the interface is modeled using a Neumann boundary condition and is based on the Fourier's law of heat diffusion which connects the gradient of temperature to the interfacial heat flux:

$-\left.k \frac{\partial \Theta(z, t)}{\partial z}\right|_{0, t}=\dot{q}(0, t)$

Furthermore, we assume that the heat flux can be neglected at the shear layer upper bound $(z=h)$ :

$\left.\frac{\partial \Theta(z, t)}{\partial z}\right|_{h, t}=0$.

Altogether, the heat conduction problem is defined by equations (Eqs. (20)-(23)). 


\subsubsection{Analytical solution to the heat conduction problem}

An analytical solution to the aforementioned set of equations can easily be obtained using Laplace transforms. In such a case, the temperature field inside the shear layer is given by the following equation, as used in [8]:

$\Theta(z, t)=\frac{1}{k} \int_{0}^{t} \dot{q}(0, \xi) \sqrt{\frac{\alpha}{\pi(t-\xi)}} \cdot \exp \left(\frac{-z^{2}}{4 \alpha(t-\xi)}\right) d \xi+\Theta(z, 0)$.

If we assume that the interfacial heat flux $\dot{q}$ is constant over a given timestep $\Delta t$ we observe that the interface temperature $\Theta(0, t)$ depends linearly on the interfacial heat flux $\dot{q}(0, t)$ :

$$
\begin{aligned}
\Theta(0, t) & =\frac{1}{k} \int_{0}^{t^{n-1}} \dot{q}(0, \xi) \sqrt{\frac{\alpha}{\pi(t-\xi)}}+\frac{q}{k} \int_{t^{n-1}}^{t^{n}} \sqrt{\frac{\alpha}{\pi(t-\xi)}} d \xi+\Theta(0,0) \\
& =\Theta_{a d}(0, t)+\mathscr{S}_{\Delta t}^{-1} \cdot \dot{q}(0, t) .
\end{aligned}
$$

Above, $\Theta_{a d}(0, t)$ would be the interface temperature observed if there were no sliding (that is without frictional heat production) during the time step, and $\mathscr{S}_{\Delta t}^{-1}$ is the inverse of the incremental Steklov-Poincare linear operator which to a given heat flux applied through the time step $\Delta t$ associates the corresponding temperature increase $\Theta-\Theta_{a d}$.

\subsubsection{Discrete heat problem}

Because of the fine scale in space which is used, the conduction problem (20) and (23) is approximated by an implicit scheme in time and finite differences in space. At each time $t$ and at each grid point $0>i>N$, the heat equation (20) then reduces to:

$\Theta_{i}^{n-1}=-\overline{F o}\left(\Theta_{i-1}^{n}+\Theta_{i+1}^{n}\right)+(1+2 \overline{F o}) \Theta_{i}^{n}$.

The adimensional Fourier mesh parameter $\overline{F_{0}}$ characterizes the heat diffusion inside the sliding material as a function of its thermal diffusivity $\alpha$ and discrete space and time steps, respectively, $\Delta z$ and $\Delta t$ :

$\overline{F o}=\frac{\alpha \Delta t}{\Delta z^{2}}$.

This equation is completed by Neumann boundary conditions at point $i=0$ and a zero heat flux condition at point $i=N$ :

B.C. at point $i=0: \quad \dot{q}^{n}=\beta_{T} \cdot[v]^{n} \cdot \tau^{n}$,

B.C. at point $i=N: \frac{k}{2 \Delta z}\left(\Theta_{N+1}^{n}-\Theta_{N-1}^{n}\right)=0$.

The temperature is thus finally obtained by solving a tridiagonal linear system:

$$
\begin{aligned}
& \left(\begin{array}{cccccc}
1+2 \overline{F o} & -2 \overline{F o} & & & & 0 \\
-\overline{F o} & 1+2 \overline{F o} & -\overline{F o} & & & \\
& -\overline{F o} & 1+2 \overline{F o} & -\overline{F o} & . & \\
& & & \cdot & \cdot & \\
0 & & & -\overline{F o} & 1+2 \overline{F o} & -\overline{F o} \\
0 & & & & -2 \overline{F o} & 1+2 \overline{F o}
\end{array}\right)\left(\begin{array}{c}
\Theta_{0}^{n} \\
\Theta_{1}^{n} \\
\Theta_{2}^{n} \\
\cdot \\
\Theta_{N-1}^{n} \\
\Theta_{N}^{n}
\end{array}\right) \\
& =\left(\begin{array}{c}
\Theta_{0}^{n-1}+2 \overline{F o}\left(\frac{\dot{q}^{n} \Delta z}{k}\right) \\
\Theta_{1}^{n-1} \\
\Theta_{2}^{n-1} \\
\cdot \\
\Theta_{N-1}^{n-1} \\
\Theta_{N}^{n-1}
\end{array}\right)
\end{aligned}
$$

from which we deduce the vector $\underline{\Theta}^{n}$ of temperature nodal values by

$\underline{\Theta}^{n}=\underline{A}+\dot{q}^{n} \cdot \underline{B}$.

For a given time $t^{n}$, the vectors of coefficients $\underline{A}$ and $\underline{B}$ (with $\underline{B}_{0}$ corresponding to the discrete inverse of the Steklov-Poincare operator $\mathscr{S}_{\Delta t}^{-1}$ ) are determined by solving Eq. (31) for two given values of $\dot{q}^{n}$. More precisely, we first compute an adiabatic temperature field $\Theta_{a d}^{n}$, and a frictional temperature field $\underline{\Theta}_{f r}^{n}$, which is obtained for a reference nonzero heat flux:

$\dot{q}_{a d}^{n}=0 \rightarrow \underline{\Theta}^{n}=\Theta_{a d}^{n}$,

$\dot{q}_{f r}^{n}=Y_{0} \cdot[v] \rightarrow \underline{\Theta}^{n}=\Theta_{f r}^{n}$

Then, the temperature field near the interface can be directly obtained by

$\left.\underline{\Theta}^{n}=\frac{\underline{\Theta}_{f r}^{n}-\underline{\Theta}_{a d}^{n}}{\dot{q}_{f r}^{n}}\right) \cdot \dot{q}^{n}+\underline{\Theta}_{a d}^{n}$.

Observe that the heat flux $\dot{q}^{n}$ given by (18) depends on the mechanical state of the interface. The thermal problem is thus coupled to the elastoplastic problem detailed below.

\subsection{Local elastoplastic model}

\subsubsection{Equation of motion}

Dynamic of a material point $z \in \Omega_{\text {int }}$ is determined by the equation of momentum:

$\operatorname{div}(\underline{\underline{\sigma}}(z, t))+\underline{f}(z, t)=\rho \cdot \underline{\gamma}(z, t) \quad \forall z \in \Omega_{\text {int }}$,

where $f$ stands for the volumic forces and $\underline{\gamma}$ for the acceleration.

\subsubsection{Elastoplastic law}

In the case of perfect shear, the elastoplastic law (9) reduces to:

$$
\frac{\partial \tau(z, t)}{\partial t}=2 G_{0}\left(\frac{1}{2} \frac{\partial v(z, t)}{\partial z}-\frac{\sqrt{3}}{2} \frac{\partial \varepsilon_{p}(z, t)}{\partial t}\right) .
$$

\subsubsection{Von Mises flow rule}

For perfect shear, the equivalent Von Mises stress $\sigma_{e q}(13)$ reduces to

$\sigma_{e q}=\sqrt{\frac{3}{2}}\|\underline{\underline{s}}(\underline{x}, t)\|=\sqrt{3} \cdot \tau(z, t)$.

Considering the shear layer geometry, the maximum shear stress for yielding must therefore satisfy

$\tau \leqslant \frac{Y(P, \Theta(z, t))}{\sqrt{3}}$

To model the behavior of metal when submitted to severe loading conditions, we determine the flow stress $Y$ using the SCG model (14) and (15) with unlimited kinematic hardening. At time $t^{n}$, the discrete form of the yield limit writes:

$Y^{n}(z)=Y_{0}\left(1+\beta \varepsilon_{p}^{n}(z)\right)^{\eta} \times \frac{G^{n}\left(P^{n}, \Theta^{n}(z)\right)}{G_{0}}$,

$G^{n}\left(P^{n}, \Theta^{n}\right)=G_{0}\left(1+g P^{n}-h_{\Theta}\left(\Theta^{n}-300\right)\right) \times \exp \left(-\frac{0.001 \cdot \Theta^{n}}{\Theta_{F}-\Theta^{n}}\right)$.

After implicit time discretization of the plastic flow rule, and explicitation of the yield limit, two cases may occur: 
- (a) Elastic local behavior:

$$
\begin{aligned}
& \tau^{n}-\frac{Y^{n}(z)}{\sqrt{3}} \leqslant 0, \\
& \Delta \varepsilon_{p}^{n}(z)=0, \\
& Y^{n}(z)=Y_{0}\left(1+\beta \varepsilon_{p}^{n-1}(z)\right)^{\eta} \times \frac{G^{n}\left(P^{n}, \Theta^{n}(z)\right)}{G_{0}} .
\end{aligned}
$$

In such a case, the shear stress at a given point $z$ is less than the yield shear stress (44). As the local plastic strain rate is equal to zero $\left(\dot{\varepsilon}_{p}^{n}(z)=0\right)$, no plastic flow is observed and therefore $\Delta \varepsilon_{p}^{n}(z)=0$.

- (b) Plastic local behaviour:

$$
\begin{aligned}
& \tau^{n}=\frac{Y^{n}(z)}{\sqrt{3}}, \\
& \Delta \varepsilon_{p}^{n}(z)>0, \\
& Y^{n}(z)=Y_{0}\left(1+\beta\left(\varepsilon_{p}^{n-1}(z)+\Delta \varepsilon_{p}^{n}(z)\right)\right)^{\eta} \times \frac{G^{n}\left(P^{n}, \Theta^{n}(z)\right)}{G_{0}} .
\end{aligned}
$$

In such a case, the shear stress at a given point $z$ is greater or equal to the yield shear stress $Y^{n}(z)$. Plastic deformation is determined by equating the shear stress with the yield limit (45), and $\Delta \varepsilon_{p}^{n}(z)>0$. By eliminating $Y^{n}$ in (45) and inverting (47), we may directly compute the cumulated plastic strain by

$$
\Delta \varepsilon_{p}^{n}\left(\tau^{n}, z\right)=\left(\frac{1}{\beta} \cdot\left\{\left(\frac{\tau^{n} \cdot G_{0} \sqrt{3}}{G^{n}(z) \cdot Y_{0}}\right)^{\frac{1}{\eta}}-1\right\}-\varepsilon_{p}^{n-1}(z)\right)_{+} .
$$

\subsection{Frictional interface model at the nanoscopic scale}

Last, we may also consider the nanoscopic scale to model the frictional behavior of the interface. The constitutive model for the frictional interface is based on the works of Oden et al. [1,2] or of Molinari [14]. It introduces a third scale associated to microscopic asperities and junctions at the sliding interface (see Fig. 11).

These junctions are of average height $\delta$ and are in contact with the hard body on a fraction $\mathbf{f}$ of the apparent contact surface $\mathbf{S}$. In order to have equilibrium of interface shear forces exerced on a substrate of area $\mathbf{S}$, the shear stress $\tau_{a}$ inside a junction is related to the shear stress $\tau$ inside the shear layer through:

$F_{T}=\tau_{a} \cdot(\mathbf{f} \cdot \mathbf{S})=\tau \cdot \mathbf{S}$.

Following Molinari [14], the elastic strain inside the junctions are neglected, which means that the plastic strain inside the junction is equal to the ratio between the relative sliding velocity $[v]$ (difference of velocity between the top and the bottom of the asperity, the top being in contact with the hard material and the bottom with the soft material), and the junction height $\delta$ :

$\dot{\varepsilon}_{p, a}=\frac{[v]}{\delta}$.

The friction force is then determined by the Coulomb-Tresca law:

$\tau_{a} \leqslant \min \left\{\mu \sigma_{n}, \frac{Y_{a}}{\sqrt{3}}\right\}$

$\left(\tau_{a}-\min \left\{\mu \sigma_{n}, \frac{Y_{a}}{\sqrt{3}}\right\}\right) \cdot[v]=0$.

Finally, the interfacial yield limit is again given by a SCG model [11]:

$$
\begin{aligned}
& Y_{a}(t)=\min \left(Y_{\max , a}, Y_{0, a}\left(1+\beta \varepsilon_{p, a}(t)\right)^{\eta}\right) \times \frac{G(P, \Theta(0, t))}{G_{0}} \\
& G(P, \Theta)=G_{0}\left(1+g P-h_{\Theta}(\Theta-300)\right) \times \exp \left(-\frac{0.001 \cdot \Theta}{\Theta_{F}-\Theta}\right) .
\end{aligned}
$$

\subsection{Mathematical characteristics of the thermomechanical problem}

From a mathematical point of view, two major unilateral constraints appear at local scale:

- First, the interface temperature $\Theta(0, t)$ must stay below the melting temperature $\Theta_{F}$.

- Furthermore, relative sliding can only occur if the interface $(z=0)$ is plastic and vice-versa. Therefore, one must respect the variational inequality defined by Eqs. (51) and (52).

Both these conditions can only be respected by the numerical model if the local problem is solved by a fully coupled time implicit scheme.

As specified, the local model is still ill posed. A boundary condition is missing in the local equation of motion. This is where the global model will influence the local one. Altogether, we therefore need two coupling conditions between the global and the local model in order to have a well posed coupled system, one in velocity, one in force.

\section{Velocity driven downscaling}

\subsection{Coupling strategy}

In a first coupling strategy, we assume that the relative sliding velocity $[v]$ at the interface is given by the hydrocode so that a coupling condition is

$[v]^{n}=\mathbb{V}_{-}^{n}(0)-\mathbb{V}_{+}^{n}(0)=[\mathbb{V}]^{n}$,

where $\mathbb{V}_{-}^{n}(0)$ is an extrapolation at point $z=0$ of the velocity computed by the hydrocode at the interface of the softer cell (see Fig. 2). We also assume that the interface is fully in contact $(f=1)$ and that its yield limit is given by the soft material yield limit (40). We finally neglect all inertia terms inside the shear layer which from the momentum equation (36) implies that the shear stress is uniform in the shear layer.

Velocity downscaling clearly simplifies the resolution of the local model and allows us to compute the shear stress $\tau(t)$ within the shear layer. This shear stress is then passed to the global model for an explicit updating of the macroscopic velocities.

\subsection{Local solver}

To compute the thermomechanical slip response at the interface, we use a simple predictor-corrector scheme which is required to integrate the governing equations (18), (35)-(37) and (48). The friction law (51)-(54) is not used in the model, since we assume sliding and since we identify the interface yield limit to the internal yield limit. We must check aposteriori that the predicted interface shear stress is at the yield limit (40). The resolution of this thermomechanical local problem consists in determining the shear stress $\tau^{n}$ (uniform from (36)) that satisfies the nonlinear elastoplastic law (37) with $\Theta$ given by (18) and (35), and $\Delta \varepsilon_{p}$ given by (48). This is done by finding a zero to the nonlinear function $\mathscr{F}\left(\tau^{n}\right)$ which is defined by

$\mathscr{F}\left(\tau^{n}\right):=h\left(\frac{\tau^{n}-\tau^{n-1}}{\Delta t}\right)+G_{0} \sqrt{3} \int_{0}^{h} \frac{\Delta \varepsilon_{p}\left(\tau^{n}, \Theta\left(\tau^{n}\right)\right)}{\Delta t} \mathrm{~d} z$.

It should be noted that this equation depends implicitly on the temperature field by means of the plastic flow rule (48). The temperature is an explicit function of velocity jump and friction through (18) and (35). Therefore, Eq. (56) is indeed a scalar equation in $\tau$ to be solved by the following secant's method: 
Velocity driven downscaling - resolution algorithm

Impose $[v]^{n}=[\mathbb{V}]^{n}$ according to equation (55)

Compute $\underline{\Theta}_{a d}$ and $\underline{\Theta}_{f r}$ using (31), (33) and (34)

Guess a predicted value $\tau_{\text {pred }}^{n}$ by the secant method

Deduce the interfacial heat flow $\dot{q}^{n}\left(\tau_{\text {pred }}^{n}\right)$ by (18),

Compute the temperature field $\Theta^{n}\left(z, \tau_{\text {pred }}^{n}\right)$

for each point $z$ by using (35),

Compute the plastic flow $\Delta \varepsilon_{p}^{n}\left(z, \tau_{\text {pred }}^{n}\right)$

for each point $z$ by using (48),

Compute $\mathcal{F}\left(\tau_{\text {pred }}^{n}\right)$ defined by (56) :

- If $\mathcal{F}\left(\tau_{\text {pred }}^{n}\right) \neq 0$, compute a new value $\tau_{\text {pred }}^{n}$ and test the loop again

- If $\mathcal{F}\left(\tau_{\text {pred }}^{n}\right)=0$, leave the loop with $\tau^{n}=\tau_{\text {pred }}^{n}$,

$$
\varepsilon_{p}^{n}=\varepsilon_{p}^{n}\left(z, \tau_{\text {pred }}^{n}\right), \Theta^{n}=\Theta^{n}\left(z, \tau_{\text {pred }}^{n}\right)
$$

\subsection{Numerical results}

The following results where obtained for a shot between aluminium and steel. The pressure was supposed to be uniform in space and constant over time. The slip velocity was also assumed to be constant in time.

The material properties considered in these computation are defined by the following set of parameters:

- For the aluminium central cone (soft material), mass density $\rho_{0}=2703 \mathrm{~kg} / \mathrm{m}^{3}$, shear modulus $G_{0}=27.6 \mathrm{GPa}$, initial tensile strength $Y_{0}=200 \mathrm{MPa}$, adimensional work hardening parameters used in the SCG model $b=125, \eta=0.10, g=6.52 \mathrm{e}^{-11}$ $\mathrm{Pa}^{-1}$ and $h_{\Theta}=6.16 \mathrm{e}^{-4} \mathrm{~K}^{-1}$, melting temperature $\Theta_{F}=1220 \mathrm{~K}$, thermal conductivity $k=117 \mathrm{~W} / \mathrm{m} \mathrm{K}$, specific heat $c=900$ $\mathrm{J} / \mathrm{kg} \mathrm{K}$ and thermal diffusivity $\alpha=48.9 \mathrm{e}^{-6} \mathrm{~m}^{2} / \mathrm{s}$.

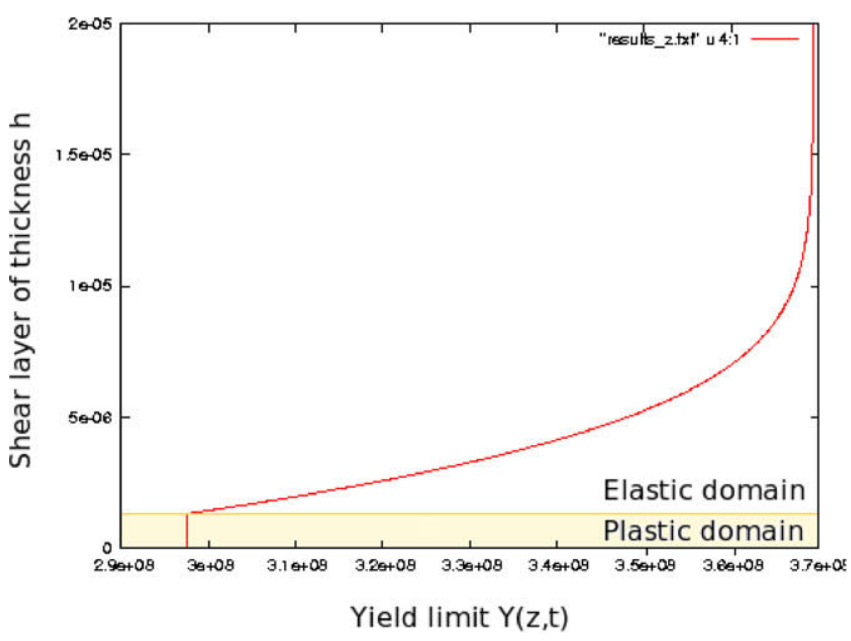

Fig. 12. Yield limit in the shear layer at a given time $t$ (thickness in meters and yield limit in Pascal).

- For the steel confinement (hard material), $\rho_{0}=7900 \mathrm{~kg} / \mathrm{m}^{3}$, $G_{0}=77 \mathrm{GPa}, Y_{0}=340 \mathrm{MPa}, b=43, \eta=0.35, g=2.26 \mathrm{e}^{-11} \mathrm{~Pa}^{-1}$, $h_{\Theta}=4.55 \mathrm{e}^{-4} \Theta^{-1}, \Theta_{F}=2380 \mathrm{~K}, k=16.1 \mathrm{~W} / \mathrm{m} \mathrm{K}, c=500 \mathrm{~J} / \mathrm{kg} \mathrm{K}$ and $\alpha=4.076 \mathrm{e}^{-6} \mathrm{~m}^{2} / \mathrm{s}$.

Then, for this tribopair configuration the coefficient of heat partition is given by $\beta_{T}=0.68$. The load and discretisation parameters used in this simulation were $h=20 \mu \mathrm{m}, P=11.2 \mathrm{GPa},[v]=$ $100 \mathrm{~m} \mathrm{~s}^{-1}, \Delta t=1 \mathrm{e}^{-9} \mathrm{~s}, \Delta z=h / 500$.

\subsubsection{Consistent data}

Fig. 12 clearly shows that thermal softening occurs on a very thin layer of matter of thickness $h=1 \mu \mathrm{m}$. Shear deformation may be observed near the interface sub-surface producing irreversible bulk plastic deformation. On a qualitative point of view, we obtain similar results (see Figs. 13 and 14) to those obtained by Ball et al. (Fig. 5).

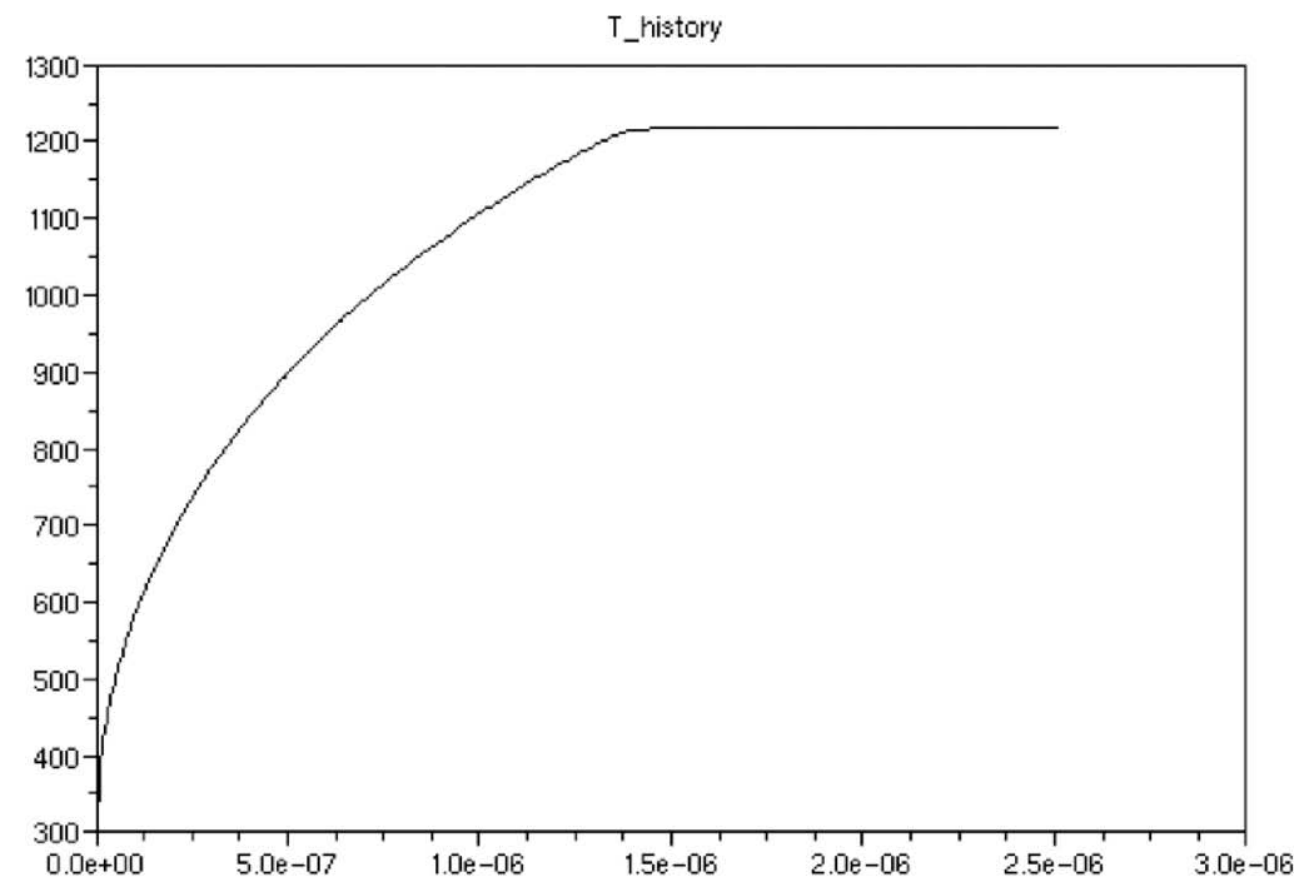

Fig. 13. Evolution with time of the interfacial temperature $\Theta(0, t)$ (time in seconds, temperature in Kelvin). 


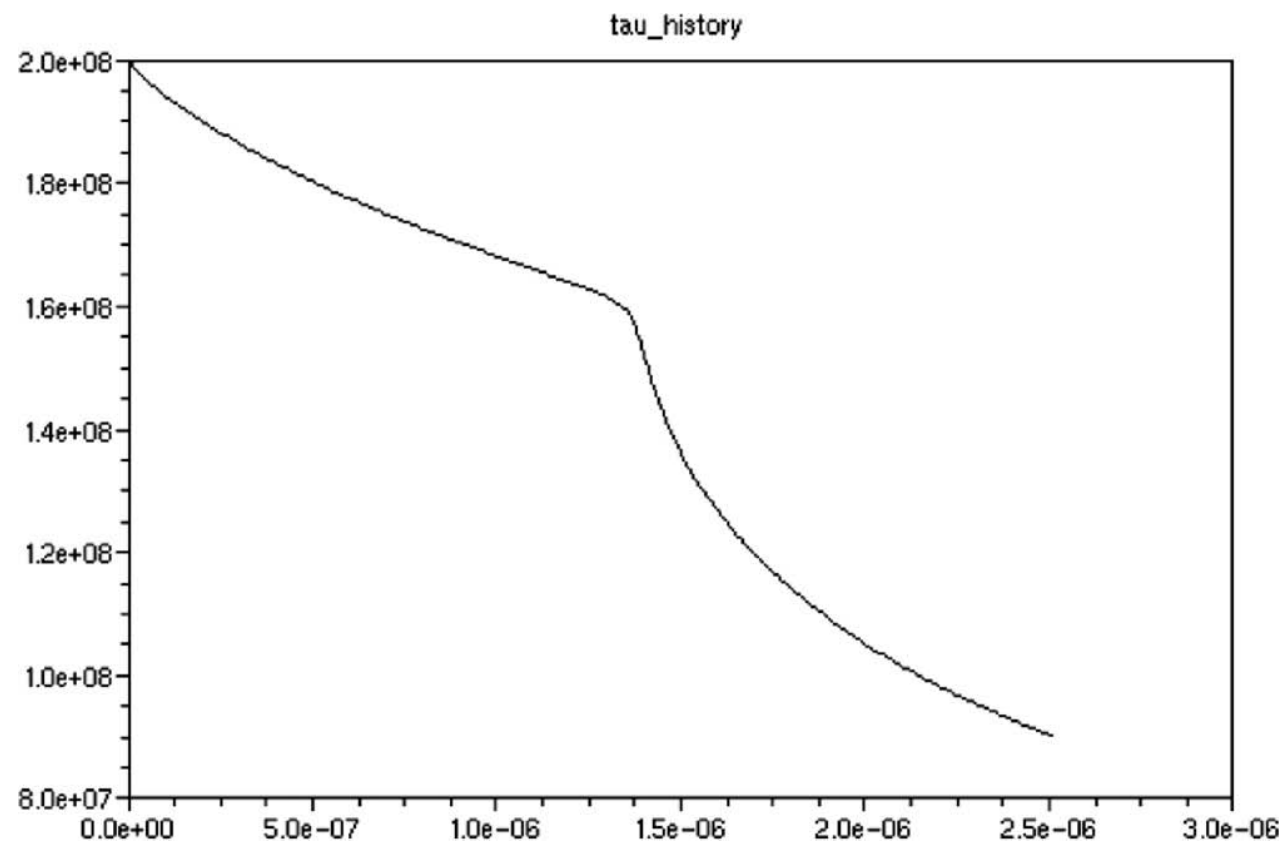

Fig. 14. Evolution with time of the interfacial shear stress $\tau(0, t)$ (time in seconds, stress in Pascal).

\subsubsection{Inconsistent data: weakness of velocity driven strategy}

Although it allows to qualitatively describe shock-driven dynamic friction, this model does not allow to take into account stick/slip phenomena. For some loading conditions described on Fig. 15, plastification of the interface brutally stops. This corresponds to elastic unloading, hence to zero plastic flow or equivalently sticking interface. But our calculation will still impose in such cases a nonzero sliding condition as inherited from the macroscopic model, violating the sliding condition imposed by (52).

On the other hand, if we relax the velocity condition, the local subproblem becomes ill posed. We must therefore develop a new strategy for imposing the velocity and shear stress coupling conditions while solving the local subproblem.

\section{Force driven downscaling}

Contrary to the previous coupling strategy, we consider herein a force driven downscaling. This coupling strategy appears to be the simplest way to obtain consistent slip velocities. In this strategy, we inherit from the macroscopic model the shear stresses at mid cell $\tau_{+}^{n}=\mathbb{T}\left(+\Delta Z / 2, t^{n}\right)$ and $\tau_{-}^{n}=\mathbb{T}\left(-\Delta Z / 2, t^{n}\right)$. The global problem on the interface cell and the local problem on the shear layer are then solved together, while respecting the coupling conditions in velocity and stresses, namely that the macroscopic and microscopic velocity $\mathbb{V}$ and $v$ are identical outside the shear layer at $z=h$ and that the macroscopic and microscopic interface shear are identical at $z=h$ so that $\mathbb{T}(h, t)=\tau(h, t)$ (see Fig. 2 ). We also

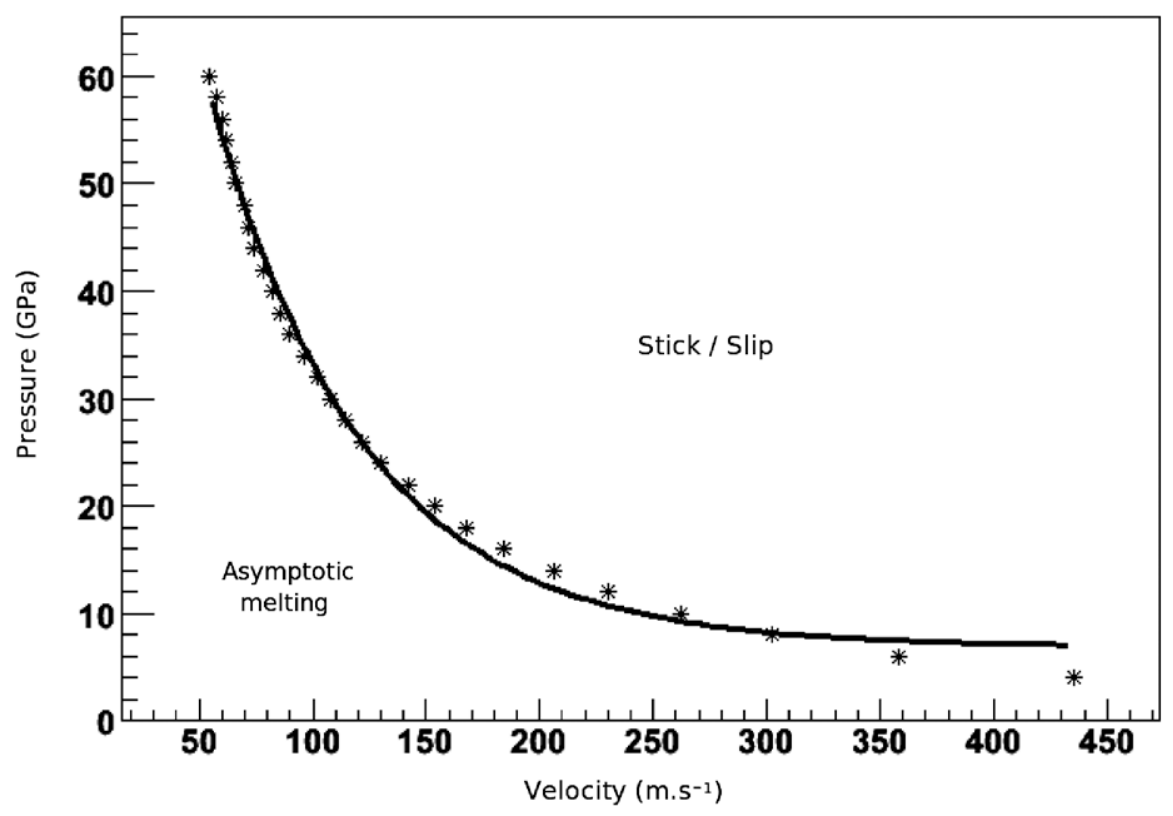

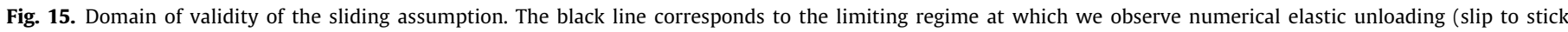
transition). 
assume that the inertia forces at macroscopic scale and at microscopic scale are identical which reduces the momentum equation (36) to the following equality:

$\frac{\partial \tau}{\partial z}=\frac{\partial \mathbb{T}}{\partial z}$.

Added to the relation $\mathbb{T}(h, t)=\tau(h, t)$, this equality implies that the macroscopic and microscopic shear stresses are identical within the shear layer.

\subsection{Global model}

At the global scale, the hydrocode solves the equation of motion using a centered scheme in time. Accelerations are computed at a given time $t^{n}$ for a given point $z$. The velocities are thus given at time $t^{n \pm \frac{1}{2}}$ by the finite difference:

$\gamma^{n}(z)=\frac{\mathbb{V}^{n+\frac{1}{2}}-\mathbb{V}^{n-\frac{1}{2}}}{\Delta t}$

In this case, writing the macroscopic conservative law on the softer material yields

$\left(\tau_{-}^{n}-\tau^{n}(0)\right) \cdot \frac{\Delta t}{m_{-}}=\left(\mathbb{V}_{-}^{n+\frac{1}{2}}(0)-\mathbb{V}_{-}^{n-\frac{1}{2}}(0)\right)$.
For the harder material and in the same way, we obtain:

$\left(\tau^{n}(0)-\tau_{+}^{n}\right) \cdot \frac{\Delta t}{m_{+}}=\left(\mathbb{V}_{+}^{n+\frac{1}{2}}(0)-\mathbb{V}_{+}^{n-\frac{1}{2}}(0)\right)$.

Subtracting Eq. (60) to Eq. (59) we have:

$\tau_{-}^{n} \frac{\Delta t}{m_{-}}+\tau_{+}^{n} \frac{\Delta t}{m_{+}}-\tau^{n}(0)\left\{\frac{\Delta t}{m_{-}}+\frac{\Delta t}{m_{+}}\right\}=[\mathbb{V}]^{n+\frac{1}{2}}-[\mathbb{V}]^{n-\frac{1}{2}}$

In the softer cell, we suppose that the macroscopic plastic strain rate $\dot{\mathbb{E}}_{p}$ is uniform in space and that the velocity field $\mathbb{V}_{-}(z, t)$ computed by the hydrocode is linear in space (see Fig. 2) such that at any point $z \in\left[0, \Delta Z_{-}\right]$, the elastoplastic constitutive law writes:

$$
\begin{aligned}
\frac{\mathrm{d} \mathbb{T}}{\mathrm{d} t}(z, t) & =G_{0}\left(\frac{\mathbb{V}_{-}\left(\Delta Z_{-}, t\right)-\mathbb{V}_{-}(0, t)}{\Delta Z_{-}}\right)-\sqrt{3} G_{0} \cdot \dot{\mathbb{E}}_{p} \\
& =G_{0}\left(\frac{\mathbb{V}_{-}(h, t)-\mathbb{V}_{-}(0, t)}{h}\right)-\sqrt{3} G_{0} \cdot \dot{\mathbb{E}}_{p} .
\end{aligned}
$$

\subsection{Coupling condition}

As defined previously, we consider at the local scale a thin layer of thickness $h \in\left[0, \Delta Z_{-}\right]$. The local velocity field is represented by $v_{-}(z, t)$ and the real relative sliding velocity is given by $[v](t)=$

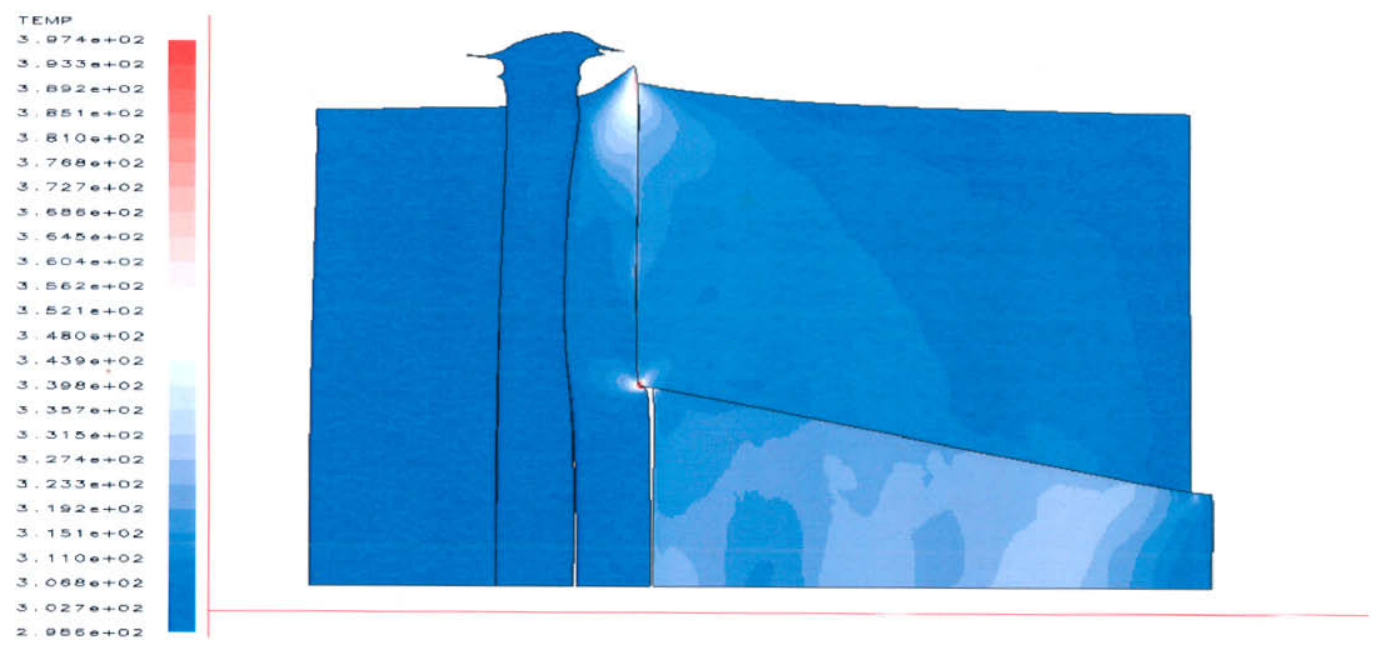

Fig. 16. Slip response of the tribopair computed by the hydrocode only at $t=12 \mu \mathrm{s}$ : shape and temperature field. No interfacial heating is observed.
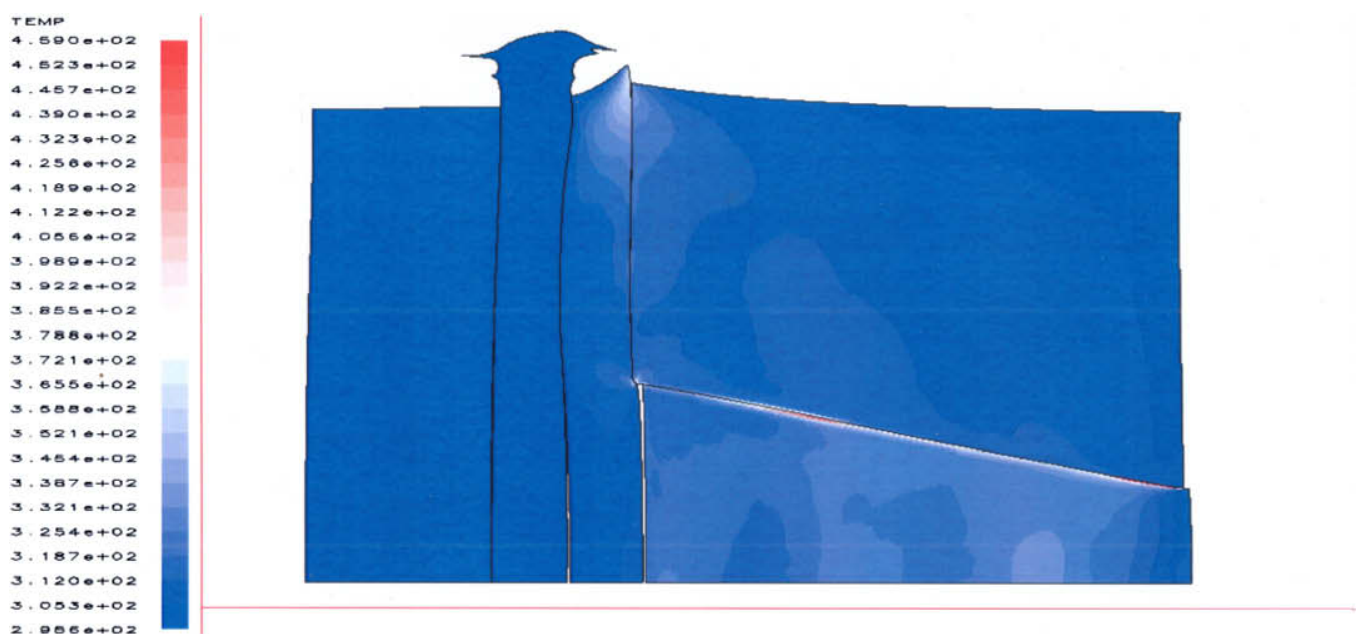

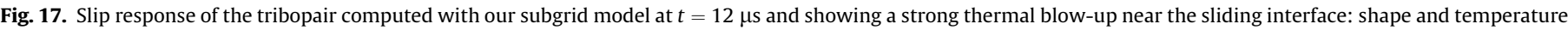
field. 
$v_{-}(0, t)-\mathbb{V}_{+}(0, t)$. We have assumed that $v_{-}(h, t)=\mathbb{V}_{-}(h, t)$ (see Fig. 2), so that the velocity field is continuous at point $z=h$.

By now, subtracting the microscopic elastoplastic constitutive law (37) to the macroscopic formulation (62), we get after integration in space,

$\frac{1}{h} \int_{0}^{h}\left[\frac{\mathrm{d} \mathbb{T}}{\mathrm{d} t}(z, t)-\frac{\mathrm{d} \tau}{\mathrm{d} t}(z, t)\right] \mathrm{d} z$
$\quad=G_{0}\left(\frac{v_{-}(0, t)-\mathbb{V}_{-}(0, t)}{h}\right)-G_{0} \sqrt{3} \cdot \dot{\mathbb{E}}_{p}+\frac{G_{0} \sqrt{3}}{h} \int_{0}^{h} \frac{\partial \varepsilon_{p}(z, t)}{\partial t} \mathrm{~d} z$.

But, by assumption, the microscopic stress and macroscopic stress are identical at each point $z$ and thus (63) reduces to

$[v](t)=[\mathbb{V}](t)-\sqrt{3} h \cdot \dot{\mathbb{E}}_{p}+\sqrt{3} \int_{0}^{h} \frac{\partial \varepsilon_{p}(z, t)}{\partial t} \mathrm{~d} z$.

The relation takes into account the coupling condition in force (63), the local conservation of momentum and the local microscopic constitutive law (37).

The local problem then reduces to the thermal equations (18) and (35), to the coupling condition (64), to the yield plastic flow rule (48) and to the friction law (51)-(54).

\subsection{Local simplified model}

For the sake of simplicity, we first consider a simple case where plastic deformation is neglected at both scales, such that $\dot{\varepsilon}_{p}(z, t)=$ $0 \forall z \in[0, h]$ implying $[v]=[\mathbb{V}]$ from (64), with $[\mathbb{V}]=\left([\mathbb{V}]^{n+\frac{1}{2}}+\right.$ $\left.[\mathbb{V}]^{n-\frac{1}{2}}\right) / 2$ given from the global model by (61). We also take $\mathbf{f}=1$ and $\delta=0$ in the friction law, which thus reduces to:

$$
\begin{aligned}
& \tau^{n} \leqslant \min \left\{\mu \sigma_{n}, \frac{Y_{\max }}{\sqrt{3}} \cdot \frac{G\left(\Theta^{n}(0)\right)}{G_{0}}\right\}, \\
& {[v]^{n} \cdot\left(\tau^{n}-\min \left\{\mu \sigma_{n}, \frac{Y_{\max }}{\sqrt{3}} \cdot \frac{G\left(\Theta^{n}(0)\right)}{G_{0}}\right\}\right)=0 .}
\end{aligned}
$$

The equilibrium equation (36) has been used in the derivation of (64). The remaining discrete local equations are given again by the thermal equations (18) and (35). It reduces to a scalar nonlinear equation in temperature:

$\underline{\Theta}^{n}=\mathscr{S}_{\Delta t}^{-1}\left(\beta_{T} \cdot[v]\left(\Theta^{n}(0)\right) \cdot \tau\left(\Theta^{n}(0)\right)\right)$

with $\tau$ given by (65) and (66) and $[v]=[\mathbb{V}]$ given by (61).

As for velocity downscaling, the thermomechanical problem defined by Eq. (67) is solved by the secant method driving to zero the residual $\mathscr{F}\left(\Theta^{n}\right)$ :

$\left.\mathscr{F}\left(\underline{\Theta}^{n}\right):=\underline{\Theta}^{n}-\underline{\Theta}_{a d}^{n}-\frac{\underline{\Theta}_{f r}^{n}-\underline{\Theta}_{a d}^{n}}{\dot{q}_{f r}^{n}}\right) \cdot \beta_{T} \cdot[v]^{n}\left(\underline{\Theta}^{n}\right) \cdot \tau^{n}\left(\underline{\Theta}^{n}\right)$.

\section{Force driven downscaling - resolution algorithm}

Compute $\underline{\Theta}_{a d}$ and $\underline{\Theta}_{f r}$ using (31), (33) and (34)

Slip test:

$$
\mid \begin{aligned}
& \text { Set }[v]^{n}=0, \\
& \text { Get } \tau^{n} \text { by }(61) \\
& \text { - If } \tau^{n} \leq \min \left\{\mu \sigma_{n}, \frac{Y_{\max }}{\sqrt{3}} \cdot \frac{G\left(\Theta_{a d}^{n}(0)\right)}{G_{0}}\right\} \text { accept }[v]=0, \\
& \Theta^{n}(0)=\Theta_{a d}^{n}(0) \text { and } \tau^{n} \text { as predicted by }(61) \\
& \text { - Else, }
\end{aligned}
$$

Guess a predicted value $\Theta_{\text {pred }}^{n}$ by the secant method

Deduce the interfacial shear stress $\tau^{n}\left(\Theta_{\text {pred }}^{n}\right)$ by (65),

Compute the relative sliding velocity $[v]^{n}\left(\Theta_{\text {pred }}^{n}\right)$ by (61)

Compute the residual $\mathcal{F}\left(\Theta_{\text {pred }}^{n}\right)$ defined by (68):

- If $\mathcal{F}\left(\Theta_{\text {pred }}^{n}\right) \neq 0$, compute a new value $\Theta_{\text {pred }}^{n}$ and test the loop again - If $\mathcal{F}\left(\Theta_{\text {pred }}^{n}\right)=0$, leave the loop with $\Theta^{n}=\Theta_{\text {pred }}^{n}, \tau^{n}=\tau^{n}\left(z, \Theta_{\text {pred }}^{n}\right)$

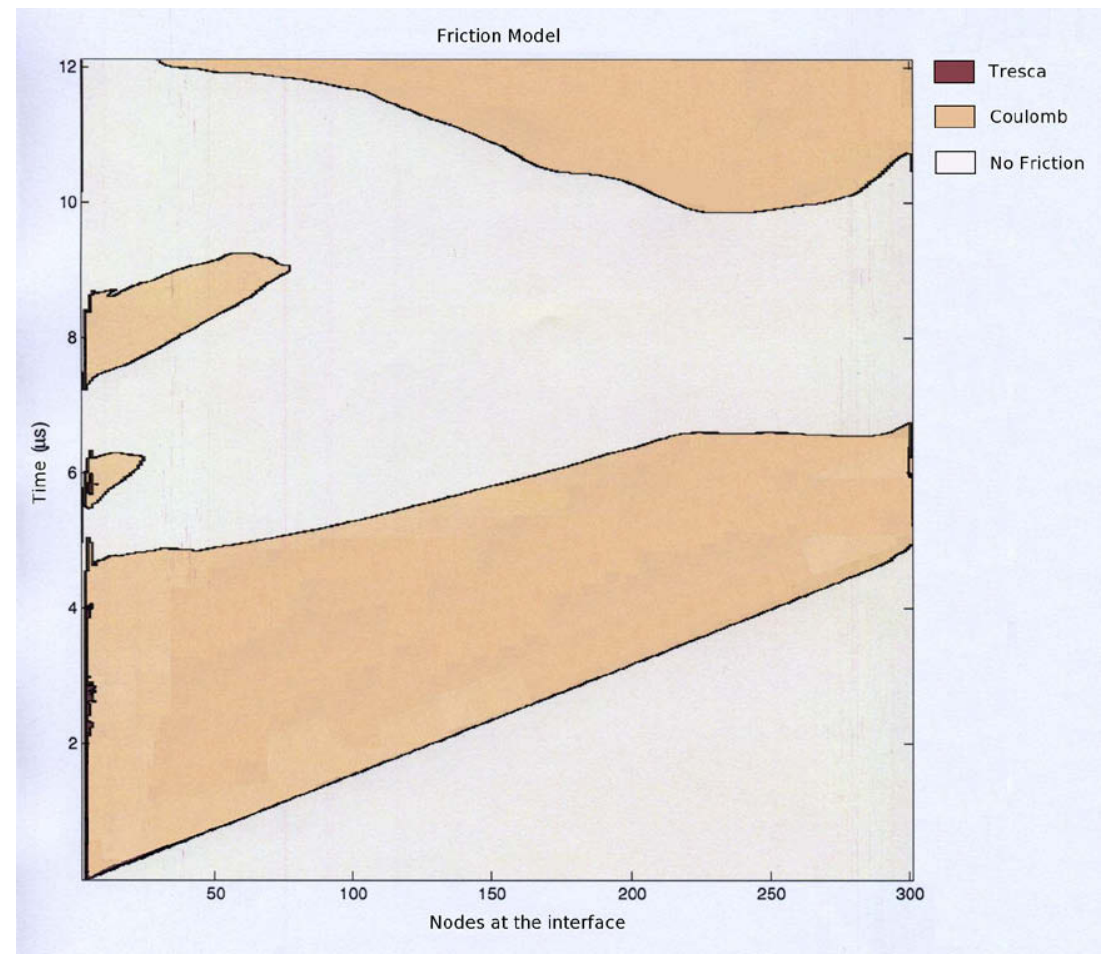

Fig. 18. Friction models used during the computation with the hydrocode only. 


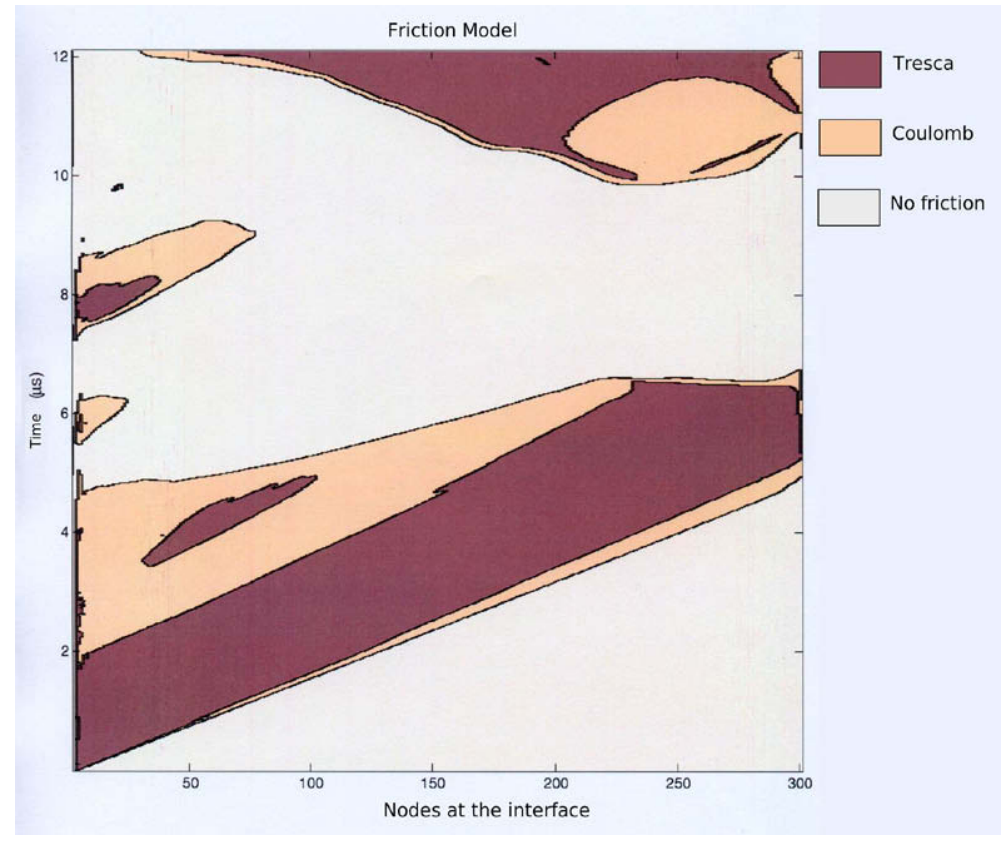

Fig. 19. Friction models used during the computation with our subgrid model.

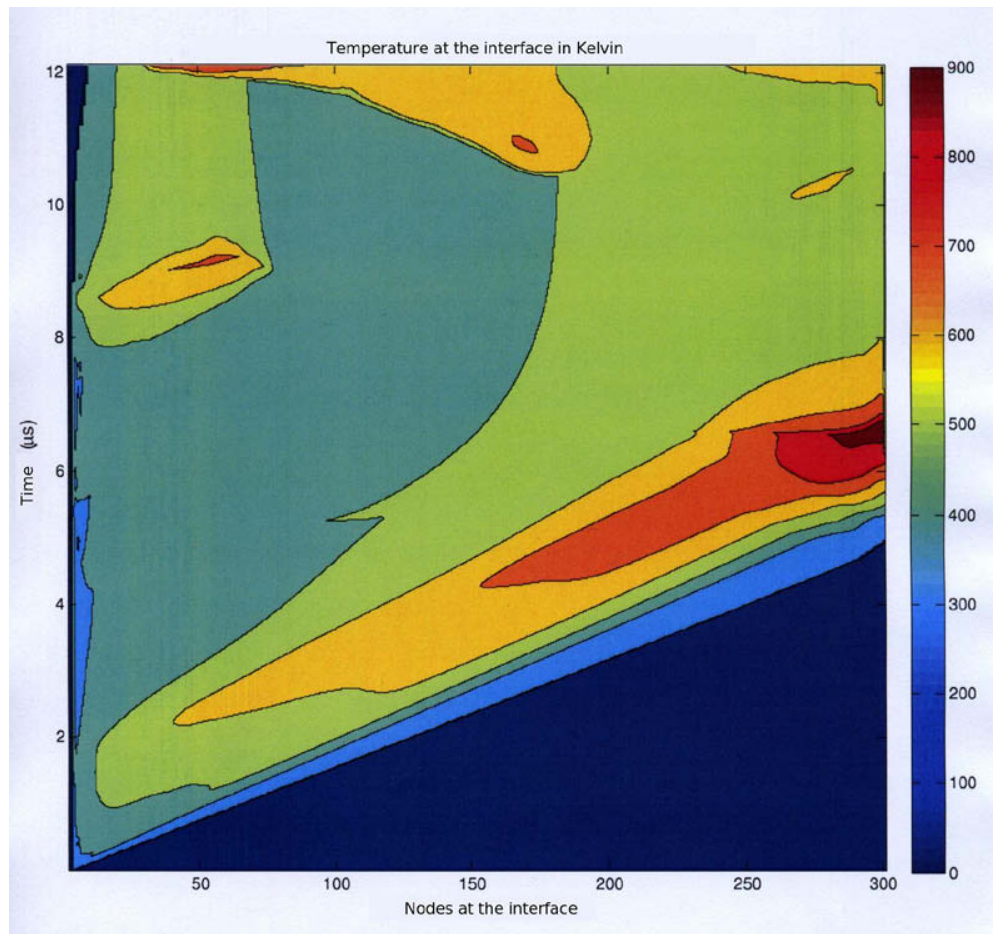

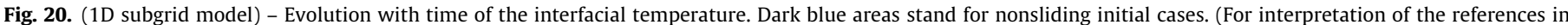
color in this figure legend, the reader is referred to the web version of this article.)

It is to note that if we take into account the plastic strain, the solution algorithm stays the same except for the equation in velocity which is now (64) with $[\mathbb{V}]$ again given by (61) and plastic flow given by (48).

\subsection{Numerical results}

The following results were obtained for shock-driven friction of an aluminium-steel tribopair. They essentially give a qualitative overview of the role played by our subgrid model on the sliding response. Therefore, we here compare the results computed by the hydrocode with or without our 1D subgrid model.

The macroscopic slip response of the tribopair is represented both on Figs. 16 and 17. In the first case, the results were computed with the hydrocode only with friction law (5). The values used in the modified Coulomb law of friction are chosen so that $\mu_{0}=0.3$, $\mu_{\infty}=0.02, \vartheta=0.01$ and $\mu_{T}=1$. This empirical model uses a very low friction coefficient $\mu_{\infty}$ in presence of large sliding velocity. 


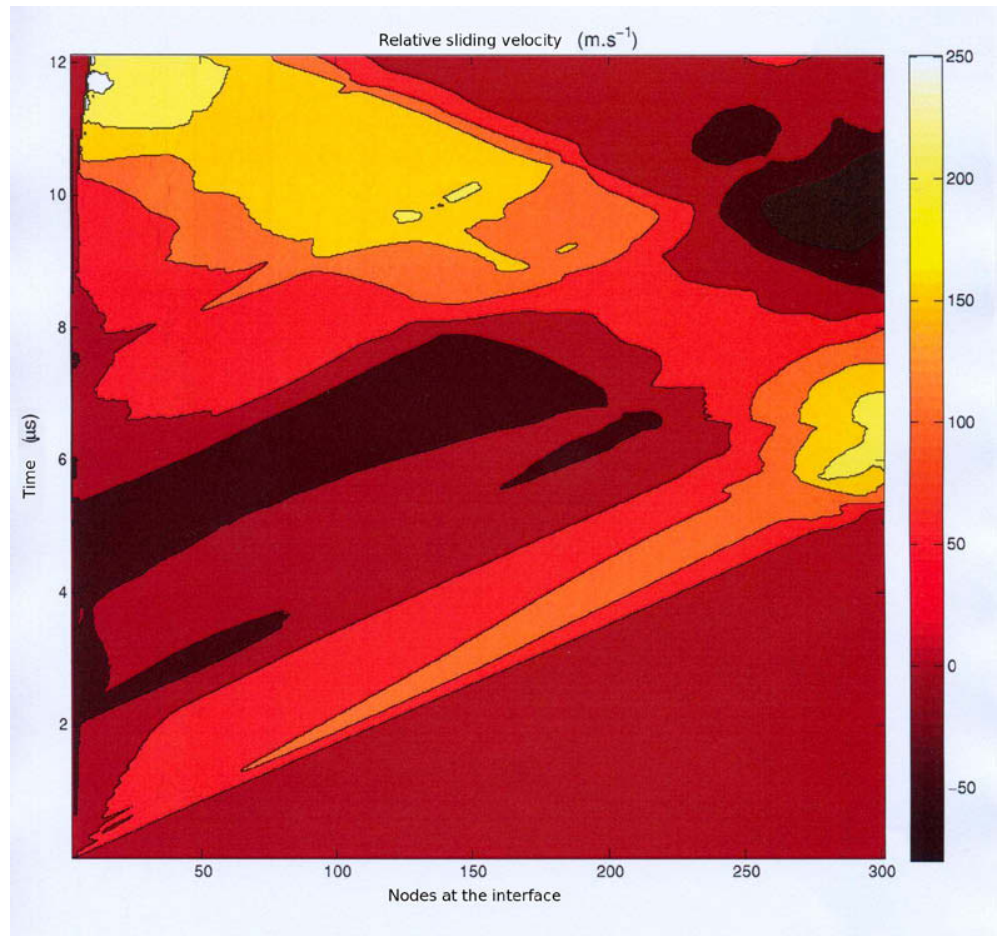

Fig. 21. (1D subgrid model) - Evolution with time of the relative sliding velocity.

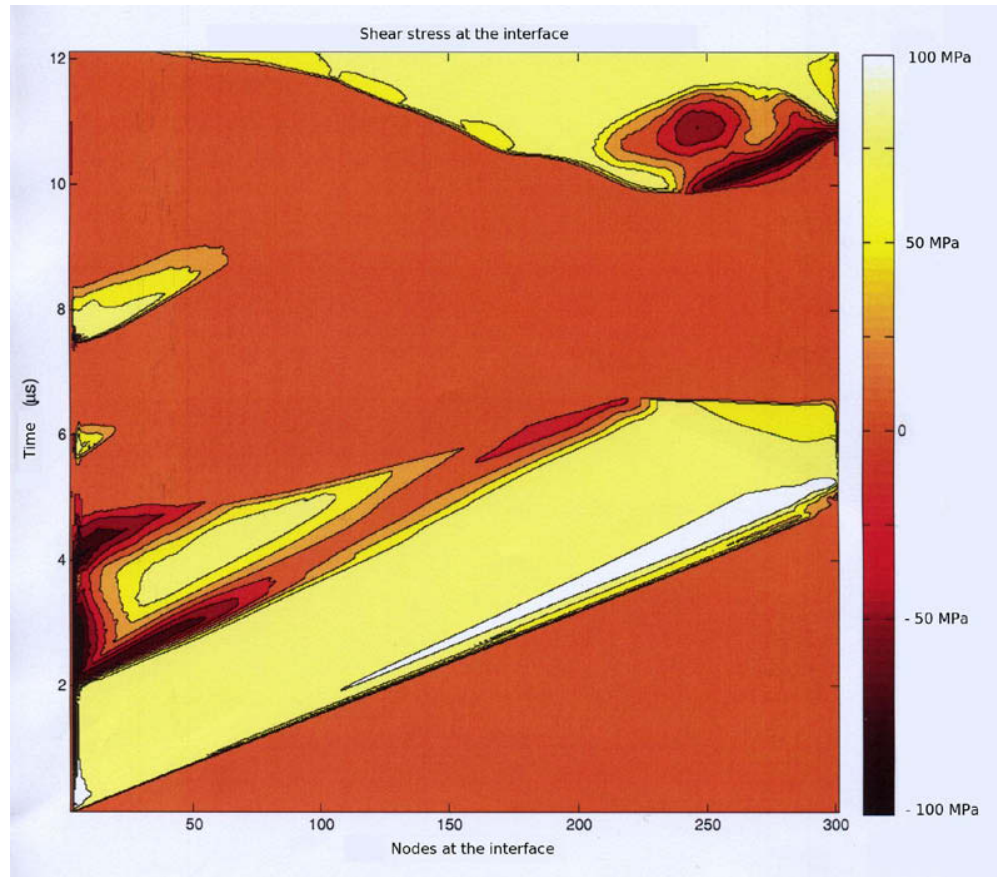

Fig. 22. (1D subgrid model) - Evolution with time of the interfacial shear stress.

As the mesh cells fail to capture the thermomechanical history at the interface, we do not observe any interfacial heating (see Fig. 16). It is not the case anymore when using our 1D subgrid model. As pictured on Figs. 17 and 20, we clearly observe a thermal blow-up located near the sliding interface, with high values of temperature. This brief overview allows us to confirm that the subgrid model has been correctly taken into account.

We also notice that due to our choice of friction coefficients and to the history of shear waves propagating inside the domain, the 1D subgrid model predicts much more friction than expected by the classical computation. Indeed we observe less sliding on Fig. 17 (1D subgrid model) than on Fig. 16 (hydrocode only).

To confirm the role played by our subgrid model, we pictured out the evolution with time of the relative sliding velocity, the interfacial shear stress, as well as the interfacial temperature. All these values have been computed at every new time step and for each interfacial point (see Figs. 18, 20-22). In both the cases 
(hydrocode only/1D subgrid model), we first determined which friction model was used.

As shown on Fig. 18, because of the very small value of $\mu_{\infty}$, the classical computation without our 1D subgrid model uses mainly the Coulomb friction model.

This result slightly differs when using our 1D subgrid model (see Figs. 19-22). In such a case, we observe much more Tresca friction, so that:

$\mu \sigma_{n} \geqslant \frac{Y_{0} \cdot G(\Theta(0, t))}{\sqrt{3}}$.

The area that represents the Tresca friction law is surrounded by the area standing for Coulomb friction. Due to thermal softening, the shear force reaches the Tresca yield stress.

\section{Conclusions}

A 1D dynamic friction model has been developed for use in hydrocodes. It provides a full coupling between heat equation, friction and plasticity and is treated at low cost through a subgrid model. Two coupling strategy have been used:

- The first case is based on velocity driven downscaling. It adequately describes the evolution of the interfacial shear stress and predicts asymptotic melting, as observed by Ball et al. [7]. Nevertheless it does not take into account stick/slip phenomena at the sliding interface.

- To avoid this physical problem a second coupling strategy based on force driven downscaling has been used. In a simplified case, the interfacial shear stress is chosen so that no plastic deformation may occur. The computed results show that the 1D model is correctly coupled to the hydrocode and adequately describe the thermal blow-up at the interface.
Further studies will be needed to correctly specify the mechanical data of the experimental problem and to take into account the plastic deformation at the interface and dynamic effects at the local scale.

\section{References}

[1] J.A.C. Martins, J.T. Oden, F.M.F. Simoes, A study of static and kinetic friction, Int. J. Engrg. Sci. 28 (1) (1990) 29-92.

[2] J.A.C. Martins, J.T. Oden, Models and computation methods for dynamic friction phenomena, Comput. Method Appl. Mech. Engrg. 52 (1985) 527-634.

[3] F.P. Bowden, E.H. Freitag, The friction of solids at very high speeds. I: Metal on metal; II: Metal on diamond, Proc. R. Soc. Lond. A248 (1958) 350-367.

[4] F.P. Bowden, P.A. Persson, Deformation, heating and melting of solids in highspeed friction, Proc. R. Soc. Lond. Ser. A: Math. Phys. Sci. 260 (1303) (1961) 433-458.

[5] R.S. Montgomery, Friction and wear at high sliding speeds, Wear 36 (3) (1976) 275-298.

[6] A. Juanicotena, Experimental investigation of dynamic friction at high contact pressure applied to an aluminium/stainless steel tribopair, J. Phys. IV, Eurodymat 2006 (2006) 559-564

[7] R.E. Winter, G.J. Ball, P.T. Keightley, Mechanisms of shock-induced dynamic friction, J. Phys. D: Appl. Phys. 39 (2006) 5043-5053.

[8] Mohammad A. Irfan, Vikas Prakash, Time resolved friction during dry sliding of metal on metal, Int. J. Solid Struct. 37 (2000) 2859-2882.

[9] N.S. Liou, M. Okada, M.A. Irfan, V. Prakash, Transient thermo-mechanical interactions during high-speed slip at metal-on-metal interfaces, Opt. Lasers Engrg. 40 (2003) 393-437.

[10] N.S. Liou, M. Okada, V. Prakash, Formation of molten films during metal-onmetal slip under extreme interfacial conditions, J. Mech. Phys. Solid 52 (2004) 2025-2056.

[11] D.J. Steinberg, C.M. Lund, A constitutive model for strain rates from $10^{-4}$ to $10^{-6} \mathrm{~s}^{-1}$, J. Appl. Phys. 65 (1989) 1528-1533.

[12] F. Armero, J. Park, An analysis of strain localization in a shear layer under thermally coupled dynamics conditions. Part 1: Continuum thermoplastic models, Int. J. Numer. Method Engrg. 56 (2003) 2069-2100.

[13] P. Vernotte, Thermocinétique Générale, Publications Scientifiques et Techniques du Ministère de l'air, No. 379, Paris, 1961.

[14] A. Molinari, Y. Estrin, S. Mercier, Dependence of the coefficient of friction on the sliding conditions in the high velocity range, J. Tribol. 121 (1999) $35-41$. 